\title{
Attentional Modulation of Hierarchical Speech Representations in a Multitalker Environment
}

\author{
Ibrahim Kiremitçi ${ }^{1,2}$, Özgür Yilmaz ${ }^{2,3}$, Emin Çelik ${ }^{1,2}$, Mo Shahdloo ${ }^{2,4}$, \\ Alexander G. Huth ${ }^{5,6,7}$ and Tolga Çukur ${ }^{1,2,3,7}$
}

\begin{abstract}
${ }^{1}$ Neuroscience Program, Sabuncu Brain Research Center, Bilkent University, Ankara TR-06800, Turkey, ${ }^{2}$ National Magnetic Resonance Research Center (UMRAM), Bilkent University, Ankara TR-06800, Turkey, ${ }^{3}$ Department of Electrical and Electronics Engineering, Bilkent University, Ankara TR-06800, Turkey, ${ }^{4}$ Department of Experimental Psychology, Wellcome Centre for Integrative Neuroimaging, University of Oxford, Oxford OX3 9DU, UK, ${ }^{5}$ Department of Neuroscience, The University of Texas at Austin, Austin, TX 78712, USA, ${ }^{6}$ Department of Computer Science, The University of Texas at Austin, Austin, TX 78712, USA and ${ }^{7}$ Helen Wills Neuroscience Institute, University of California, Berkeley, CA 94702, USA

Address correspondence to Ibrahim Kiremitçi, National Magnetic Resonance Research Center (UMRAM), Bilkent University, Ankara TR-06800, Turkey. Email: i.kiremitci@bilkent.edu.tr. Tolga Çukur, Department of Electrical and Electronics Engineering, Room 304, Bilkent University, Ankara TR-06800, Turkey. Email: cukur@ee.bilkent.edu.tr
\end{abstract}

\begin{abstract}
Humans are remarkably adept in listening to a desired speaker in a crowded environment, while filtering out nontarget speakers in the background. Attention is key to solving this difficult cocktail-party task, yet a detailed characterization of attentional effects on speech representations is lacking. It remains unclear across what levels of speech features and how much attentional modulation occurs in each brain area during the cocktail-party task. To address these questions, we recorded whole-brain blood-oxygen-level-dependent (BOLD) responses while subjects either passively listened to single-speaker stories, or selectively attended to a male or a female speaker in temporally overlaid stories in separate experiments. Spectral, articulatory, and semantic models of the natural stories were constructed. Intrinsic selectivity profiles were identified via voxelwise models fit to passive listening responses. Attentional modulations were then quantified based on model predictions for attended and unattended stories in the cocktail-party task. We find that attention causes broad modulations at multiple levels of speech representations while growing stronger toward later stages of processing, and that unattended speech is represented up to the semantic level in parabelt auditory cortex. These results provide insights on attentional mechanisms that underlie the ability to selectively listen to a desired speaker in noisy multispeaker environments.
\end{abstract}

Key words: cocktail-party, dorsal and ventral stream, encoding model, fMRI, natural speech

\section{Introduction}

Humans are highly adept at perceiving a target speaker in crowded multispeaker environments (Shinn-Cunningham and Best 2008; Kidd and Colburn 2017; Li et al. 2018). Auditory attention is key to behavioral performance in this difficult "cocktailparty problem" (Cherry 1953; Fritz et al. 2007; McDermott 2009; Bronkhorst 2015; Shinn-Cunningham et al. 2017). Literature consistently reports that attention selectively enhances cortical responses to the target stream in auditory cortex and beyond, while filtering out nontarget background streams (Hink and Hillyard 1976; Teder et al. 1993; Alho et al. 1999, 2003, 2014; Jäncke et al. 2001, 2003; Lipschutz et al. 2002; Rinne et al. 2008; Rinne 2010; Elhilali et al. 2009; Gutschalk and Dykstra 2014). However, the precise link between the response modulations and underlying speech representations is less clear. Speech representations are hierarchically organized across multiple 
stages of processing in cortex, with each stage selective for diverse information ranging from low-level acoustic to highlevel semantic features (Davis and Johnsrude 2003; Griffiths and Warren 2004; Hickok and Poeppel 2004, 2007; Rauschecker and Scott 2009; Okada et al. 2010; Friederici 2011; Di Liberto et al. 2015; de Heer et al. 2017; Brodbeck et al. 2018a). Thus, a principal question is to what extent attention modulates these multilevel speech representations in the human brain during a cocktail-party task (Miller 2016; Simon 2017).

Recent electrophysiology studies on the cocktail-party problem have investigated attentional response modulations for natural speech stimuli (Kerlin et al. 2010; Ding and Simon 2012a, 2012b; Mesgarani and Chang 2012; Power et al. 2012; Zion Golumbic et al. 2013; Puvvada and Simon 2017; Brodbeck et al. 2018b; O'Sullivan et al. 2019; Puschmann et al. 2019). Ding and Simon (2012a, 2012b) fit spectrotemporal encoding models to predict cortical responses from the speech spectrogram. Attentional modulation in the peak amplitude of spectrotemporal response functions was reported in planum temporale in favor of the attended speech. Mesgarani and Chang (2012) built decoding models to estimate the speech spectrogram from responses measured during passive listening and examined the similarity of the decoded spectrogram during a cocktail-party task to the isolated spectrograms of attended versus unattended speech. They found higher similarity to attended speech in nonprimary auditory cortex. Zion Golumbic et al. (2013) reported amplitude modulations in speech-envelope response functions toward attended speech across auditory, inferior temporal, frontal, and parietal cortices. Other studies using decoding models have similarly reported higher decoding performance for the speech envelope of the attended stream in auditory, prefrontal, motor, and somatosensory cortices (Puvvada and Simon 2017; Puschmann et al. 2019). Brodbeck et al. (2018b) further identified peak amplitude response modulations for sublexical features including word onset and cohort entropy in temporal cortex. Note that because these electrophysiology studies fit models for acoustic or sublexical features, the reported attentional modulations primarily comprised relatively low-level speech representations.

Several neuroimaging studies have also examined wholebrain cortical responses to natural speech in a cocktail-party setting (Nakai et al. 2005; Alho et al. 2006; Hill and Miller 2010; Ikeda et al. 2010; Wild et al. 2012; Regev et al. 2019; Wikman et al. 2021). In the study of Hill and Miller (2010), subjects were given an attention cue (attend to pitch, attend to location or rest) and later exposed to multiple speech stimuli where they performed the cued task. Partly overlapping frontal and parietal activations were reported, during both the cue and the stimulus exposure periods, as an effect of attention to pitch or location in contrast to rest. Furthermore, pitch-based attention was found to elicit higher responses in bilateral posterior and right middle superior temporal sulcus, whereas location-based attention elicited higher responses in left intraparietal sulcus. In alignment with electrophysiology studies, these results suggest that attention modulates relatively low-level speech representations comprising paralinguistic features. In a more recent study, Regev et al. (2019) measured responses under 2 distinct conditions: while subjects were presented bimodal speech-text stories and asked to attend to either the auditory or visual stimulus, and while subjects were presented unimodal speech or text stories. Intersubject response correlations were measured between unimodal and bimodal conditions. Broad attentional modulations in response correlation were reported from primary auditory cortex to temporal, parietal, and frontal regions in favor of the attended modality. Although this finding raises the possibility that attention might also affect representations in higher-order regions, a systematic characterization of individual speech features that drive attentional modulations across cortex is lacking.

An equally important question regarding the cocktail-party problem is whether unattended speech streams are represented in cortex despite the reported modulations in favor of the target stream (Bronkhorst 2015; Miller 2016). Electrophysiology studies on this issue identified representations of low-level spectrogram and speech envelope features of unattended speech in early auditory areas (Mesgarani and Chang 2012; Ding and Simon 2012a, 2012b; Zion Golumbic et al. 2013; Puvvada and Simon 2017; Brodbeck et al. 2018b; Puschmann et al. 2019), but no representations of linguistic features (Brodbeck et al. 2018b). Meanwhile, a group of neuroimaging studies found broader cortical responses to unattended speech in superior temporal cortex (Scott et al. 2004, 2009a; Wild et al. 2012; Scott and McGettigan 2013; Evans et al. 2016; Regev et al. 2019). Specifically, Wild et al. (2012) and Evans et al. (2016) reported enhanced activity associated with the intelligibility of unattended stream in parts of superior temporal cortex extending to superior temporal sulcus. Although this implies that responses in relatively higher auditory areas carry some information regarding unattended speech stimuli, the specific features of unattended speech that are represented across the cortical hierarchy of speech is lacking.

Here we investigated whether and how attention affects representations of attended and unattended natural speech across cortex. To address these questions, we systematically examined multilevel speech representations during a diotic cocktail-party task using naturalistic stimuli. Whole-brain BOLD responses were recorded in 2 separate experiments (Fig. 1) while subjects were presented engaging spoken narratives from "The Moth Radio Hour." In the passive-listening experiment, subjects listened to single-speaker stories for over $2 \mathrm{~h}$. Separate voxelwise models were fit that measured selectivity for spectral, articulatory, and semantic features of natural speech during passive listening (de Heer et al. 2017). In the cocktail-party experiment, subjects listened to temporally overlaid speech streams from 2 speakers while attending to a target category (male or female speaker). To assess attentional modulation in functional selectivity, voxelwise models fit during passive listening were used to predict responses for the cocktail-party experiment. Model performances were calculated separately for attended and unattended stories. Attentional modulation was taken as the difference between these 2 performance measurements. Comprehensive analyses were conducted to examine the intrinsic complexity and attentional modulation of multilevel speech representations and to investigate up to what level of speech features unattended speech is represented across cortex.

\section{Materials and Methods}

\section{Participants}

Functional data were collected from 5 healthy adult native subjects (4 males and one female; aged between 26 and 31) who had no reported hearing problems and were native English speakers. The experimental procedures were approved by the Committee for the Protection of Human Subjects at University of California, Berkeley. Written informed consent was obtained from all subjects. 
experiments

a. passive-listening experiment

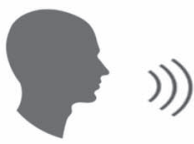

b. cocktail-party experiment

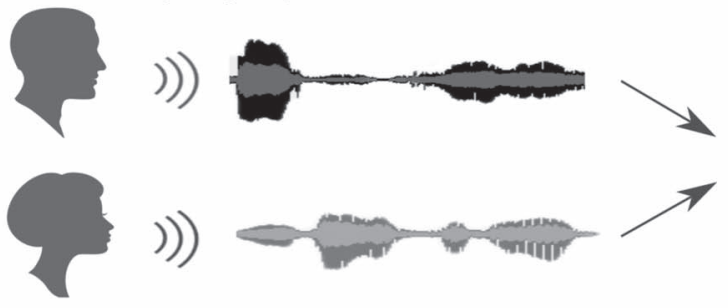

stimuli

single-speaker stimulus

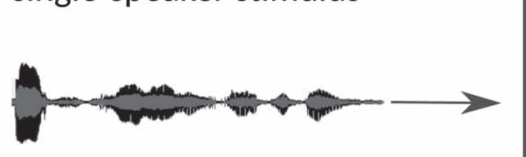

two-speaker stimulus conditions

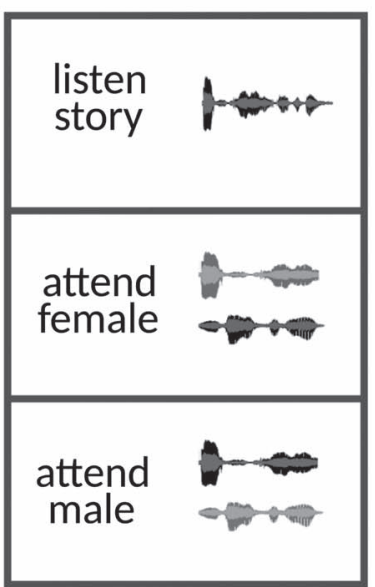

Figure 1. Experimental design. (a) "Passive-listening experiment." 10 stories from Moth Radio Hour were used to compile a single-speaker stimulus set. Subjects were instructed to listen to the stimulus vigilantly without any explicit task in the passive-listening experiment. (b) "Cocktail-party experiment." A pair of stories told by individuals of different genders were selected from the single-speaker stimulus set and overlaid temporally to generate a 2-speaker stimulus set. Subjects were instructed to attend either to the male or female speaker in the cocktail-party experiment. The same 2-speaker story was presented twice in separate runs while the target speaker was varied. Attention condition was fixed within runs and it alternated across runs.

\section{Stimuli}

Figure 1 illustrates the 2 main types of stimuli used in the experiments: single-speaker stories and 2-speaker stories. Ten singlespeaker stories were taken from The Moth Radio Program: "Alternate Ithaca Tom" by Tom Weiser; "How to Draw a Nekkid Man" by Tricia Rose Burt; "Life Flight" by Kimberly Reed; "My Avatar and Me" by Laura Albert; "My First Day at the Yankees" by Matthew McGough; "My Unhurried Legacy" by Kyp Malone; "Naked" by Catherine Burns; "Ode to Stepfather" by Ethan Hawke; "Targeted" by Jen Lee; and "Under the Influence" by Jeffery Rudell. All stories were told before a live audience by a male or female speaker, and they were about 10-15 min long. Each 2-speaker story was generated by temporally overlaying a pair of stories told by different genders and selected from the single-speaker story set. When the durations of the 2 single-speaker stories differed, the longer story was clipped from the end to match durations. Three 2-speaker stories were prepared: from "Targeted" and "Ode to Stepfather" (cocktail1); from "How to Draw a Nekkid Man" and "My First Day at the Yankees" (cocktail2); and from "Life Flight" and "Under the Influence" (cocktail3). In the end, the stimuli consisted of 10 single-speaker and three 2-speaker stories.

\section{Experimental Procedures}

Figure 1 outlines the 2 main experiments conducted in separate sessions: passive-listening and cocktail-party experiments. In the passive-listening experiment, subjects were instructed to listen to single-speaker stories vigilantly without an explicit attentional target. To facilitate sustained vigilance, we picked engaging spoken narratives from the Moth Radio Hour (see Stimuli). Each of the 10 single-speaker stories was presented once in a separate run of the experiment. Two 2-hour sessions were conducted, resulting in 10 runs of passive-listening data for each subject. In the cocktail-party experiment, subjects were instructed to listen to 2-speaker stories while attending to a target speaker (either the male or the female speaker). Our experimental design focuses on attentional modulations of speech representations when a stimulus is attended versus unattended. Each of the 3 cocktail-stories was presented twice in separate runs. This allowed us to present the same stimulus set in attended and unattended conditions to prevent potential biases due to across condition stimulation differences. To minimize adaptation effects, different 2-speaker stories were presented in consecutive runs while maximizing the time window between repeated presentations of a 2speaker story. Attention condition alternated across consecutive runs. An exemplary sequence of runs was: cocktail1-M (attend to male speaker in cocktail1), cocktail2-F (attend to female speaker in cocktail2), cocktail3-M, cocktail1-F, cocktail2$\mathrm{M}$, and cocktail3-F. The first attention condition assigned to each 2-speaker story ( $\mathrm{M}$ or $\mathrm{F}$ ) was counterbalanced across subjects. This resulted in a balanced assignment of "attended" versus "unattended" conditions during the second exposure to each 2 speaker story. Furthermore, for each subject, the second exposure to half of the single-speaker stories (3 out of 6 included within the 2-speaker stories) coincided with the "attended" condition, whereas the second exposure to the other half coincided with the "unattended" condition. Hence, second exposure to each story was balanced across "attended" and "unattended" conditions both within and across subjects. A 2-hour session was conducted, resulting in 6 runs of cocktail-data. Note that the 2-speaker stories used in the cocktail-party experiment were constructed from the single-speaker story set used in passivelistening experiment. Hence, for each subject, the cocktail-party experiment was conducted several months ( $\sim 5.5$ months) after the completion of the passive-listening experiment to minimize potential repetition effects. The dataset collected from the passive-listening experiment was previously analyzed (Huth et al. 2016; de Heer et al. 2017); however, the dataset collected from the cocktail-party experiment was specifically collected for this study. 
In both experiments, the length of each run was tailored to the length of the story stimulus with additional $10 \mathrm{~s}$ of silence both before and after the stimulus. All stimuli were played at $44.1 \mathrm{kHz}$ and delivered binaurally to both ears using Sensimetrics S14 in-ear piezo-electric headphones. The Sensimetrics S14 is a magnetic resonance imaging (MRI)-compatible auditory stimulation system with foam canal tips to reduce scanner noise (above $29 \mathrm{~dB}$ as stated in specifications). The frequency response of the headphones was flattened using a Behringer Ultra-Curve Pro Parametric Equalizer. Furthermore, the level of sound was adjusted for each subject to ensure clear and comfortable hearing of the stories.

\section{MRI Data Collection and Preprocessing}

MRI data were collected on a 3T Siemens TIM Trio scanner at the Brain Imaging Center, UC Berkeley, using a 32-channel volume coil. For functional scans, a gradient echo EPI sequence was used with $\mathrm{TR}=2.0045 \mathrm{~s}, \mathrm{TE}=31 \mathrm{~ms}$, flip angle $=70^{\circ}$, voxel size $=2.24$ $\times 2.24 \times 4.1 \mathrm{~mm}^{3}$, matrix size $=100 \times 100$, field of view $=224$ $\times 224 \mathrm{~mm}^{2}$ and 32 axial slices covering the entire cortex. For anatomical data, a T1-weighted multiecho MP-RAGE sequence was used with voxel size $=1 \times 1 \times 1 \mathrm{~mm}^{3}$ and field of view $=256$ $\times 212 \times 256 \mathrm{~mm}^{3}$.

Each functional run was motion corrected using FMRIB's Linear Image Registration Tool (FLIRT) (Jenkinson and Smith 2001). A cascaded motion-correction procedure was performed, where separate transformation matrices were estimated within single runs, within single sessions and across sessions sequentially. To do this, volumes in each run were realigned to the mean volume of the run. For each session, the mean volume of each run was then realigned to the mean volume of the first run in the session (see Supplementary Table 1 for within-session motion statistics during the cocktail-party experiment). Lastly, the mean volume of the first run of each session was realigned to the mean volume of the first run of the first session of the passive-listening experiment. The estimated transformation matrices were concatenated and applied in a single step. Motion-corrected data were manually checked to ensure that no major realignment errors remained. The moment-to-moment variations in head position were also estimated and used as nuisance regressors during model estimation to regress out motion-related nuisance effects from BOLD responses. The Brain Extraction Tool in FSL 5.0 (Smith 2002) was used to remove nonbrain tissues. This resulted in 68016-84 852 brain voxels in individual subjects. All model fits and analyses were performed on these brain voxels in volumetric space.

\section{Visualization on Cortical Flatmaps}

Cortical flatmaps were used for visualization purposes, where results in volumetric space were projected onto the cortical surfaces using PyCortex (Gao et al. 2015). Cortical surfaces were reconstructed from anatomical data using Freesurfer (Dale et al. 1999). Five relaxation cuts were made into the surface of each hemisphere, and the surface crossing the corpus callosum was removed. Functional data were aligned to the individual anatomical data with affine transformations using FLIRT (Jenkinson and Smith 2001). Cortical flatmaps were constructed for visualization of significant model prediction scores, functional selectivity and attentional modulation profiles, and representational complexity and modulation gradients.

\section{ROI Definitions and Abbreviations}

We defined region of interests for each subject based on an atlas-based parcellation of the cortex (Destrieux et al. 2010). To do this, functional data were coregistered to the individualsubject anatomical scans with affine transformations using FLIRT (Jenkinson and Smith 2001). Individual-subject anatomical data were then registered to the Freesurfer standard anatomical space via the boundary-based registration tool in FSL (Greve and Fischl 2009). This procedure resulted in subject-specific transformations mapping between the standard anatomical space and the functional space of individual subjects. Anatomical regions of interest from the Destrieux atlas were outlined in the Freesurfer standard anatomical space; and they were back-projected onto individual-subject functional spaces via the subject-specific transformations using PyCortex (Gao et al. 2015). The anatomical regions were labeled according to the atlas. To explore potential selectivity gradients across the lateral aspects of Superior Temporal Gyrus and Superior Temporal Sulcus, these ROIs were further split into 3 equidistant subregions in posterior-to-anterior direction. Heschl's Gyrus and Heschl's Sulcus were considered as a single ROI as prior reports suggest that primary auditory cortex is not constrained by Heschl's Gyrus and extends to Heschl's Sulcus as well (Woods et al. 2009, 2010; da Costa et al. 2011). We only considered regions with at least 10 speech-selective voxels in each individual subject for subsequent analyses.

Supplementary Table 2 lists the defined ROIs and the number of spectrally, articulatorily, and semantically selective voxels within each ROI, with number of speech-selective voxels. ROI abbreviations and corresponding Destrieux indices are Heschl's Gyrus and Heschl's Sulcus (HG/HS: 33 and 74), Planum Temporale (PT: 36), posterior segment of Slyvian Fissure (pSF: 41), lateral aspect of Superior Temporal Gyrus (STG: 34), Superior Temporal Sulcus (STS, 73), Middle Temporal Gyrus (MTG: 38), Angular Gyrus (AG: 25), Supramarginal Gyrus (SMG: 26), Intraparietal Sulcus (IPS: 56), opercular part of Inferior Frontal Gyrus/Pars Opercularis (POP: 12), triangular part of Inferior Frontal Gyrus/Pars Triangularis (PTR: 14), Precentral Gyrus (PreG: 29), medial Occipito-Temporal Sulcus (mOTS:60), Inferior Frontal Sulcus (IFS: 52), Middle Frontal Gyrus (MFG:15), Middle Frontal Sulcus (MFS: 53), Superior Frontal Sulcus (SFS: 54), Superior Frontal Gyrus (SFG: 16), Precuneus (PreC: 30), Subparietal Sulcus (SPS: 71), and Posterior Cingulate Cortex (PCC: 9 and 10). The subregions of STG are aSTG (anterior one-third of STG), mSTG (middle one-third of STG), and pSTG (posterior one-third of STG). The subregions of STS are aSTS (anterior one-third of STS), mSTS (middle one-third of STS) and pSTS (posterior one-third of STS). MTG was not split into subregions since these subregions did not have a sufficient number of speech-selective voxels in each individual subject.

\section{Model Construction}

To comprehensively assess speech representations, we constructed spectral, articulatory, and semantic models of the speech stimuli (Fig. 2; de Heer et al. 2017).

\section{Spectral Model}

For the spectral model, cochleogram features of speech were estimated based on Lyon's Passive Ear model. Lyon's human cochlear model involves logarithmic filtering, compression and adaptive gain control operations applied to input sound (Lyon 
Speech Waveform

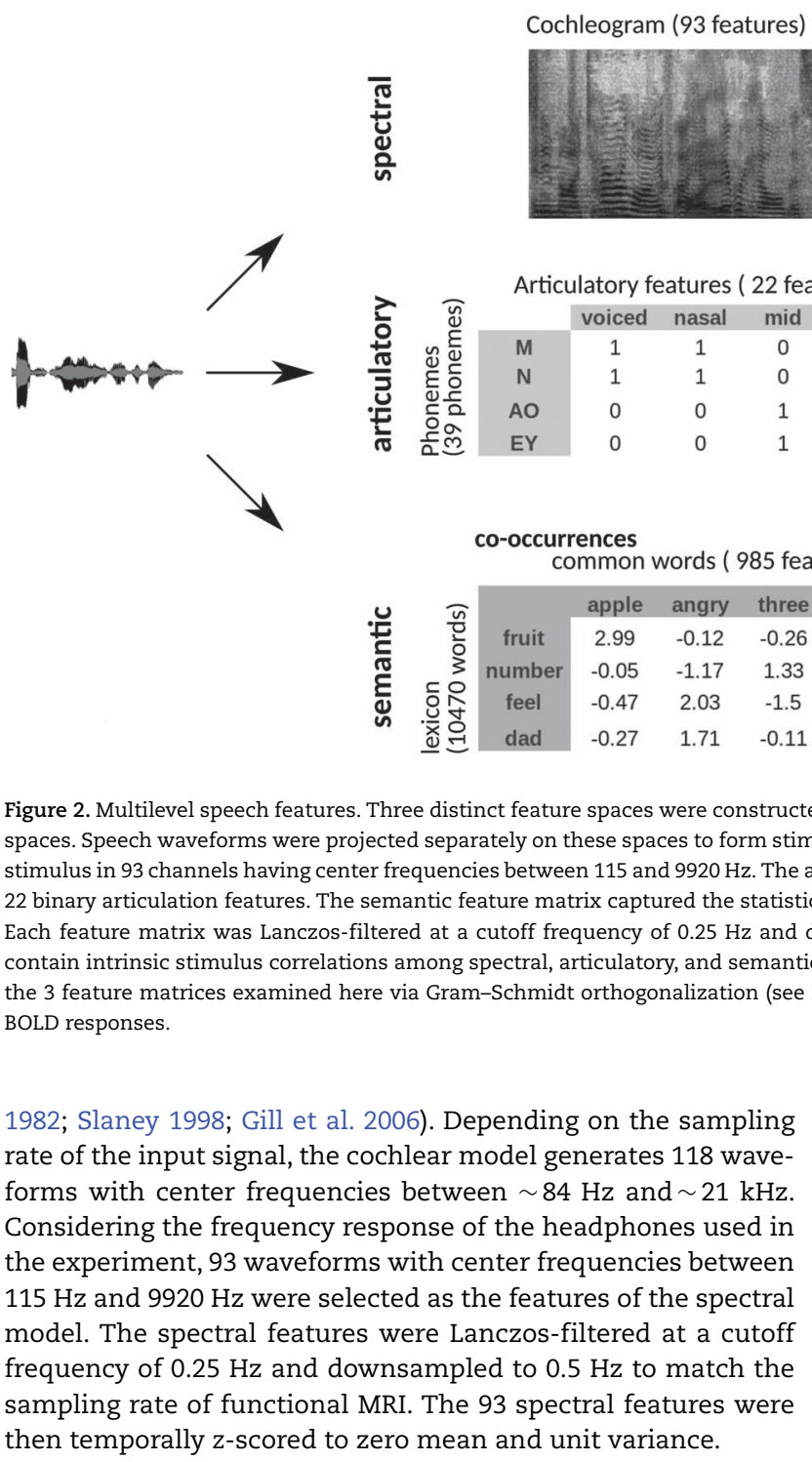

\section{Articulatory Model}

For the articulatory model, each phoneme in the stories was mapped onto a unique set of 22 articulation features; for example, phoneme $/ 3 /$ is postalveolar, fricative, and voiced (Levelt 1993; de Heer et al. 2017). This mapping resulted in 22dimensional binary vectors for each phoneme. To obtain the timestamp of each phoneme and word in the stimuli, the speech in the stories was aligned with the story transcriptions using the Penn Phonetics Lab Forced Aligner (Yuan and Liberman 2008). Alignments were manually verified and corrected using Praat (www.praat.org). The articulatory features were Lanczos-filtered at a cutoff frequency of $0.25 \mathrm{~Hz}$ and downsampled to $0.5 \mathrm{~Hz}$. Finally, the 22 articulatory features were $z$-scored to zero mean and unit variance.

\section{Semantic Model}

For the semantic model, co-occurrence statistics of words were measured via a large corpus of text (Mitchell et al. 2008; Huth et al. 2016; de Heer et al. 2017). The text corpus was compiled from 2405569 Wikipedia pages, 36333459 user comments scraped from reddit.com, 604 popular books, and the transcripts of 13 Moth stories (including the stories used as stimuli). We then built a 10470-word lexicon from the union set of the 10000 most common words in the compiled corpus and all words appearing in the 10 Moth stories used in the experiment. Basis words were then selected as a set of 985 unique words from Wikipedia's List of 1000 Basic Words. Co-occurrence statistics of the lexicon words with 985 basis words within a 15-word window were characterized as a cooccurrence matrix of size $985 \times 10470$. Elements of the resulting co-occurrence matrix were log-transformed, z-scored across columns to correct for differences in basis-word frequency, and $z$-scored across rows to correct for differences in lexicon-word frequency. Each word in the stimuli was then represented with a 985-dimensional co-occurrence vector based on the speechtranscription alignments. The semantic features were Lanczosfiltered at a cutoff frequency of $0.25 \mathrm{~Hz}$ and downsampled to $0.5 \mathrm{~Hz}$. The 985 semantic features were finally z-scored to zero mean and unit variance.

Decorrelation of Feature Spaces

In natural stories, there might be potential correlations among certain spectral, articulatory, or semantic features. If significant, 
such correlations can partly confound assessments of model performance. To assess the unique contribution of each feature space to the explained variance in BOLD responses, a decorrelation procedure was first performed (Fig. 2). To decorrelate a feature matrix $\mathrm{F}$ of size $m \times n$ from a second feature matrix $\mathrm{K}$ of size $m x p$, we first found an orthonormal basis for the column space of $\mathrm{K}(\operatorname{col}\{\mathrm{K}\})$ using economy-size singular value decomposition:

$$
K_{m x p}=U_{m x p} \times S_{p x p} \times V_{p x p}
$$

where $U$ contains left singular vectors as columns, $\mathrm{V}$ contains right singular vectors, and $S$ contains the singular values. Left singular vectors were taken as the orthonormal basis for $\operatorname{col}\{K\}$, and each column of $F$ was decorrelated from it according to the following formula:

$$
\overrightarrow{f_{i}^{d}}=\overrightarrow{f_{i}}-\sum_{j=1}^{p}\left(\overrightarrow{f_{i}} \cdot \overrightarrow{u_{j}}\right) \cdot \overrightarrow{u_{j}}
$$

where $\vec{f}_{i}, \vec{u}_{j}$ are the column vectors of $F$ and $U$ respectively, and $\overrightarrow{f_{i}^{d}}$ is the column vectors of the decorrelated feature matrix, $F^{d}$. To decorrelate feature matrices for the models considered here, we took the original articulatory feature matrix as a reference, and decorrelated the spectral feature matrix from the articulatory feature matrix, and decorrelated the semantic feature matrix from both articulatory and spectral feature matrices. This decorrelation sequence was selected because spectral and articulatory features capture lower-level speech representations, and the articulatory feature matrix had the fewest number of features among all models. In the end, we obtained 3 decorrelated feature matrices whose columns had zero correlation with the columns of the other 2 matrices.

\section{Analyses}

The main motivation of this study is to understand whether and how strongly various levels of speech representations are modulated across cortex during a cocktail-party task. To answer this question, we followed a 2-stage approach as illustrated in Figure 3. In the first stage, we identified voxels selective for speech features using data from the passive-listening experiment. To do this, we measured voxelwise selectivity separately for spectral, articulatory, and semantic features of the singlespeaker stories. In the second stage, we used the models fit using passive-listening data to predict BOLD responses measured in the cocktail-party experiment. Prediction scores for attended versus unattended stories were compared to quantify the degree of attentional modulations, separately for each model and globally across all models.

Note that a subset of the 10 single-speaker stories was used to generate three 2-speaker stories used in the experiments. To prevent potential bias, a 3-fold cross-validation procedure was performed for testing models fit using passive-listening data on cocktail-party data. In each fold, models were fit using 8-run passive-listening data; and separately tested on 2-run passivelistening data and 2-run cocktail-party data. The same set of test stories were used both in the passive-listening and cocktailparty experiments to minimize risk of poor model generalization between the passive-listening and cocktail-party experiments due to uncontrolled stimulus differences. There was no overlap between the stories in the training and testing runs. Model predictions were aggregated across 3-fold, and prediction scores were then computed.

\section{Voxelwise Modeling}

In the first stage, we fit voxelwise models in individual subjects using passive-listening data. To account for hemodynamic delays, we used a linearized 4-tap finite impulse response (FIR) filter to allow different HRF shapes for separate brain regions (Goutte et al. 2000). Each model feature was represented as 4 features in the stimulus matrix to account for their delayed effects in BOLD responses at 2, 4, 6, and 8 s. Model weights, W, were then found using L2-regularized linear regression:

$$
\mathrm{W}=\left(F^{\mathrm{T}} F+I \lambda\right)^{-1} F^{\mathrm{T}} R
$$

Here, $\lambda$ is the regularization parameter, $F$ is the decorrelated feature matrix for a given model and $R$ is the aggregate BOLD response matrix for cortical voxels. A cross-validation procedure with 50 iterations was performed to find the best regularization parameter for each voxel among 30 equispaced values in logspace of $1: 10^{5}$. The training passive-listening data was split into 50 equisized chunks, where 1 chunk was reserved for validation and 49 chunks were reserved for model fitting at each iteration. Prediction scores were taken as Pearson's correlation between predicted and measured BOLD responses. The optimal $\lambda$ value for each voxel was selected by maximizing the average prediction score across cross-validation folds. The final model weights were obtained using the entire set of training passive-listening data and the optimal $\lambda$. Next, we measured the prediction scores of the fit models on testing data from the passive-listening experiment. Spectrally, articulatorily, and semantically selective voxels were separately identified in each ROI based on the set of significantly predicted voxels by each model. A given ROI was considered selective for a model, only if it contained 10 or more significant voxels for that model $\left(\mathrm{q}(\mathrm{FDR})<10^{-5} ; \mathrm{t}-\right.$ test). Speechselective voxels within the ROI were then taken as the union of these spectrally, articulatorily, and semantically selective voxels. Subsequent analyses were performed on speech-selective voxels.

1. Model-specific selectivity index. Single-voxel prediction scores on passive-listening data were used to quantify the degree of selectivity of each ROI to the underlying model features under passive-listening. To do this, a model-specific selectivity index, $\left(\mathrm{SI}_{m}\right)$, was defined as follows:

$$
\mathrm{SI}_{m}=\frac{(r)_{m}}{\sum_{i}(r)_{i}}, \quad i, m \in\{\text { spe, art, sem }\}
$$

where $r$ is the average prediction score across speech-selective voxels within the ROI during passive-listening. $\mathrm{SI}_{m}$ is in the range of $[0,1]$, where higher values indicate stronger selectivity for the underlying model.

2. Complexity index. The complexity of speech representations was characterized via a complexity index, (CI), which 


\section{a. Voxelwise modeling}

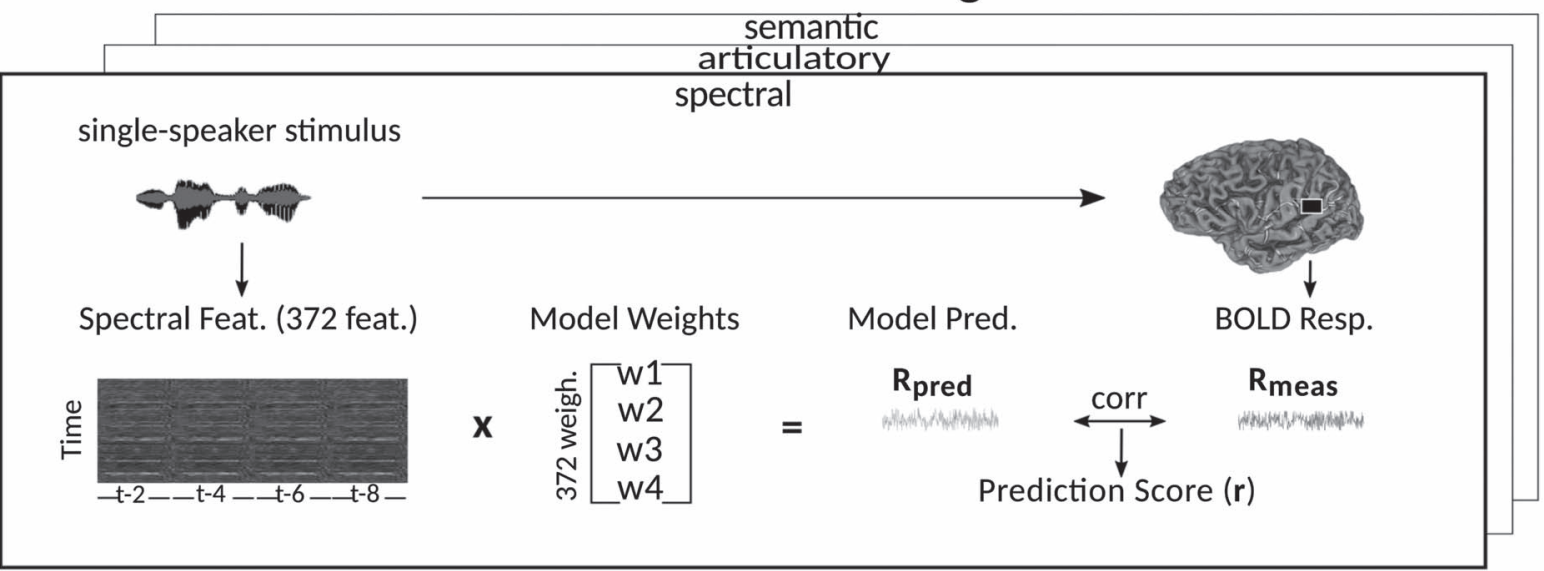

\section{b. Assessment of attentional modulation}

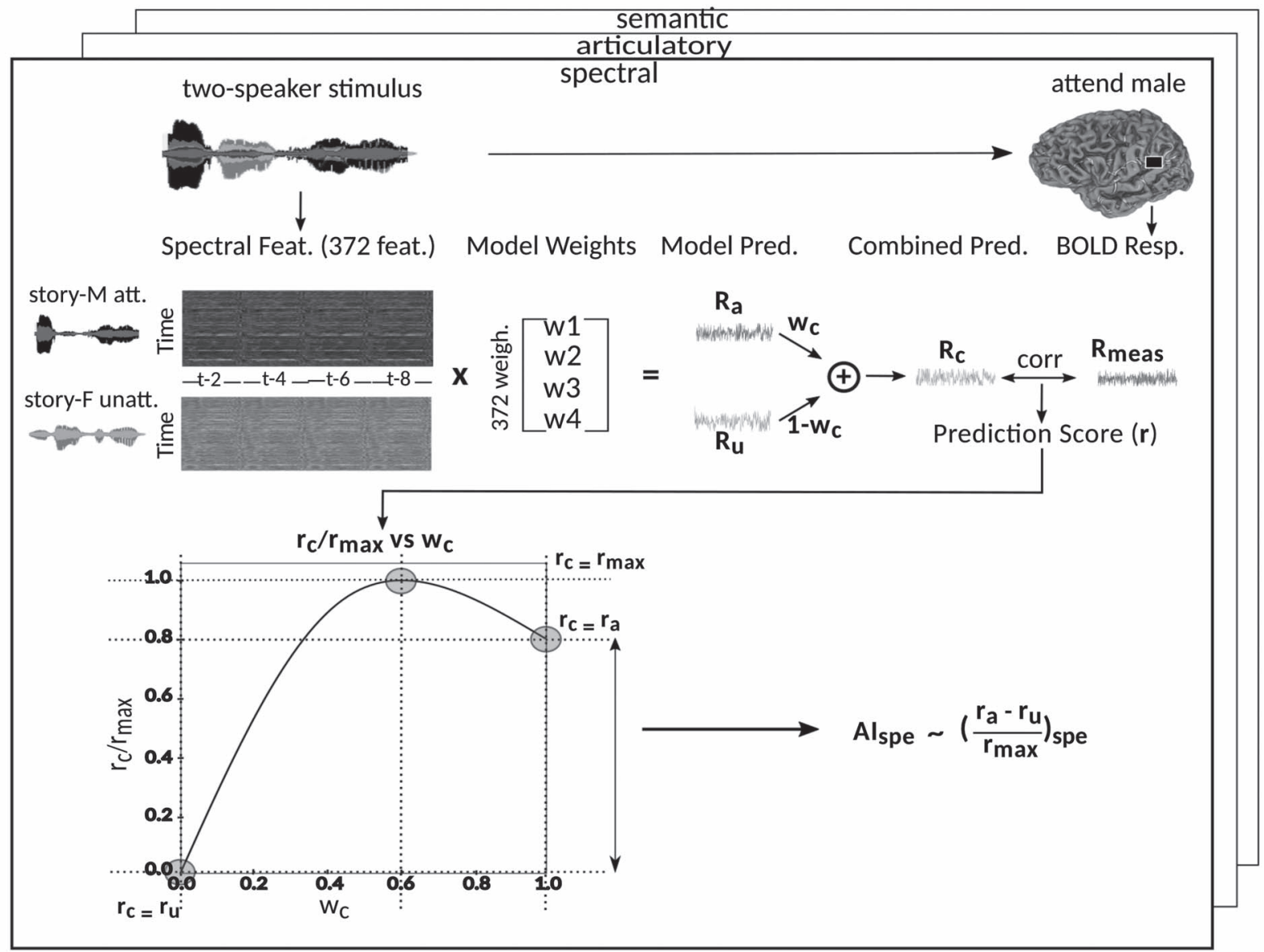

Figure 3. Modeling procedures. (a) "Voxelwise modeling." Voxelwise models were fit in individual subjects using passive-listening data. To account for hemodynamic response, a linearized 4-tap FIR filter spanning delayed effects at 2-8 s was used. Models were fit via L2-regularized linear regression. BOLD responses were predicted based on fit voxelwise models on held-out passive-listening data. Prediction scores were taken as the Pearson's correlation between predicted and measured BOLD responses. For a given subject, speech-selective voxels were taken as the union of voxels significantly predicted by spectral, articulatory, or semantic models ( $($ FDR $)<10^{-5}$, t-test). (b) "Assessment of attentional modulation." Passive-listening models for single voxels were tested on cocktail-party data to quantify attentional modulations in selectivity. In a given run, one of the speakers in a 2-speaker story was attended while the other speaker was ignored. Separate response predictions were obtained using the isolated story stimuli for the attended speaker and for the unattended speaker. Since a voxel can represent information from both attended and unattended stimuli, a linear combination of these predicted responses was considered with varying combination weights ( $\mathrm{w}_{\mathrm{c}}$ in [0 1]). BOLD responses were predicted based on each combination weight separately. Three separate prediction scores were calculated based on only the attended stimulus ( $\left.\mathrm{w}_{\mathrm{C}}=1\right)$, based on only the unattended stimulus $\left(\mathrm{w}_{\mathrm{C}}=0\right)$, and based on the optimal combination of the 2 stimuli. A model-specific attention index, $\left(\mathrm{AI}_{m}\right)$ was then computed as the ratio of the difference in prediction scores for attended versus unattended stories to the prediction score for their optimal combination (see Materials and Methods). 
reflected the relative tuning of an ROI for low- versus highlevel speech features. The following intrinsic complexity levels were assumed for the 3 speech models considered here: $\left(c_{\text {spe }}, c_{\text {art }}, c_{\text {sem }}\right)=(0.0,0.5,1.0)$. Afterward, CI was taken as the average of the complexity levels weighted by the selectivity indices:

$$
\mathrm{CI}=\sum_{m} \mathrm{SI}_{m} c_{m}, \quad m \in\{\text { spe, art, sem }\}
$$

$\mathrm{CI}$ is in the range of $[0,1]$, where higher values indicate stronger tuning for semantic features and lower values indicate stronger tuning for spectral features.

\section{Assessment of Attentional Modulations}

In the second stage, we tested the passive-listening models on cocktail-party data to quantify ROI-wise attentional modulation in selectivity for corresponding model features and to find the extent of the representation of unattended speech. These analyses were repeated separately for the 3 speech models.

1. Model-specific attention index. To quantify the attentional modulation in selectivity for speech features, we compared prediction scores for attended versus unattended stories in the cocktail-party experiment. Models fit using passivelistening data were used to predict BOLD responses elicited by 2-speaker stories. In each run, only one of the speakers in a 2-speaker story was attended, whereas the other speaker was ignored. Separate response predictions were obtained using the isolated story stimuli for the attended and unattended speakers. Since a voxel can represent information on both attended and unattended stimuli, a weighted linear combination of these predicted responses was considered:

$$
R_{c}=R_{a} w_{c}+R_{u}\left(1-w_{c}\right)
$$

Where $R_{a}$ and $R_{u}$ are the predicted responses for the attended and unattended stories in a given run; $R_{c}$ is the combined response and $w_{c}$ is the combination weight. We computed $R_{c}$ for each separate $w_{c}$ value in [0:0.1:1]. Note that $R_{c}=R_{a}$ when $w_{c}=1.0$; and $R_{c}=R_{u}$ when $w_{c}=0.0$. We then calculated single-voxel prediction scores for each $w_{c}$ value. An illustrative plot of $r_{c} / r_{\text {max }}$ versus $w_{c}$ is given in Figure $3 b$, where $r_{c}$ denotes the prediction scores and $r_{\max }$ denotes the maximum $r_{c}$ value (the optimal combination). $r_{a}$ and $r_{u}$ are the prediction scores for attended and unattended stories respectively. To quantify the degree of attentional modulation, a model-specific attention index $\left(\mathrm{AI}_{m}\right)$ was taken as:

$$
\mathrm{AI}_{m}=\alpha_{m}\left(\frac{r_{a}-r_{u}}{r_{\max }}\right)_{m}, \alpha_{m}=\frac{\left(r_{\max }\right)_{m}}{\sum_{\mathrm{i}}\left(r_{\max }\right)_{i}}, \quad m, i \in\{\text { spe, art, sem }\}
$$

where $r_{\text {max }}$ denotes an ideal upper limit for model performance, and $\alpha_{m}$ reflects the relative model performance under the cocktail-party task. Note that $\mathrm{AI}_{m}$ considers selectivity to the underlying model features when calculating the degree of attentional modulation.

2. Global attention index (gAI). We then computed gAI as follows:

$$
\mathrm{gAI}=\sum_{m} \mathrm{AI}_{m}, \quad m \in\{\text { spe, art, sem }\}
$$

Both gAI and $\mathrm{AI}_{m}$ are in the range $[-1,1]$. A positive index indicates attentional modulation of selectivity in favor of the attended stimuli and a negative index indicates attentional modulation in favor of the unattended stimuli. A value of zero indicates no modulation.

Colormap in Selectivity and Modulation Profile Flatmaps

The cortical flatmaps of selectivity and modulation profiles use a colormap that shows the relative contributions of all 3 models to the selectivity and attention profiles. For selectivity profiles, a continuous colormap was created by assigning significantly positive articulatory, semantic and spectral selectivity to the red, green and blue (R, G, B) color channels, respectively. During assignment, selectivity values were normalized to sum of one, and then normalized to linearly map the interval [0.15 0.85] to $\left[\begin{array}{ll}0 & 1\end{array}\right]$. Distinct colors were assigned to 6 landmark selectivity values: red for $(1,0,0)$, green for $(0,1,0)$, blue for $(0,0,1)$, yellow for $(0.5,0.5,0)$, magenta for $(0.5,0,0.5)$, and turquoise for $(0,0.5,0.5)$. The same procedures were also applied for creating a colormap for modulation profiles.

\section{Statistical Tests}

Significance Assessments within Subjects

For each voxel-wise model, the significance of prediction scores was assessed via a t-test; and resulting $P$ values were falsediscovery-rate corrected for multiple comparisons (FDR; Benjamini and Hochberg 1995).

A bootstrap test was used in assessments of $\mathrm{SI}_{m}, \mathrm{CI}, \mathrm{AI}_{m}$, and gAI within single subjects. In ROI analyses, speech-selective voxels within a given ROI were resampled with replacement 10000 times. For each bootstrap sample, mean prediction score of a given model was computed across resampled voxels. Significance level was taken as the fraction of bootstrap samples in which the test metric computed from these prediction scores is less than 0 (for right-sided tests) or greater than 0 (for left-sided tests). The same procedure was also used for comparing pairs of ROIs, where ROI voxels were resampled independently.

\section{Significance Assessments Across Subjects}

A bootstrap test was used in assessments of $\mathrm{SI}_{m}, \mathrm{CI}, \mathrm{AI}_{m}$, and gAI across subjects. In ROI analyses, ROI-wise metrics were resampled across subjects with replacement 10000 times. Significance level was taken as the fraction of bootstrap samples where the test metric averaged across resampled subjects is less than 0 (for right-sided tests) or greater than 0 (for left-sided tests). The same procedure was also used for comparisons among pairs of ROIs. Here, we used a more stringent significance definition for across-subjects tests that focuses on effects consistently observed in each individual subject. Therefore, an effect was taken significant only if the same metric was found significant in each individual subject.

\section{Results}

\section{Attentional Modulation of Multilevel Speech Representations}

To examine the cortical distribution and strength of attention modulations in speech representations, we first obtained a baseline measure of intrinsic selectivity for speech features. For this purpose, we fit voxelwise models using BOLD responses recorded during passive listening. We built 3 separate models containing low-level spectral, intermediate-level articulatory, and high-level semantic features of natural stories (de Heer et al. 


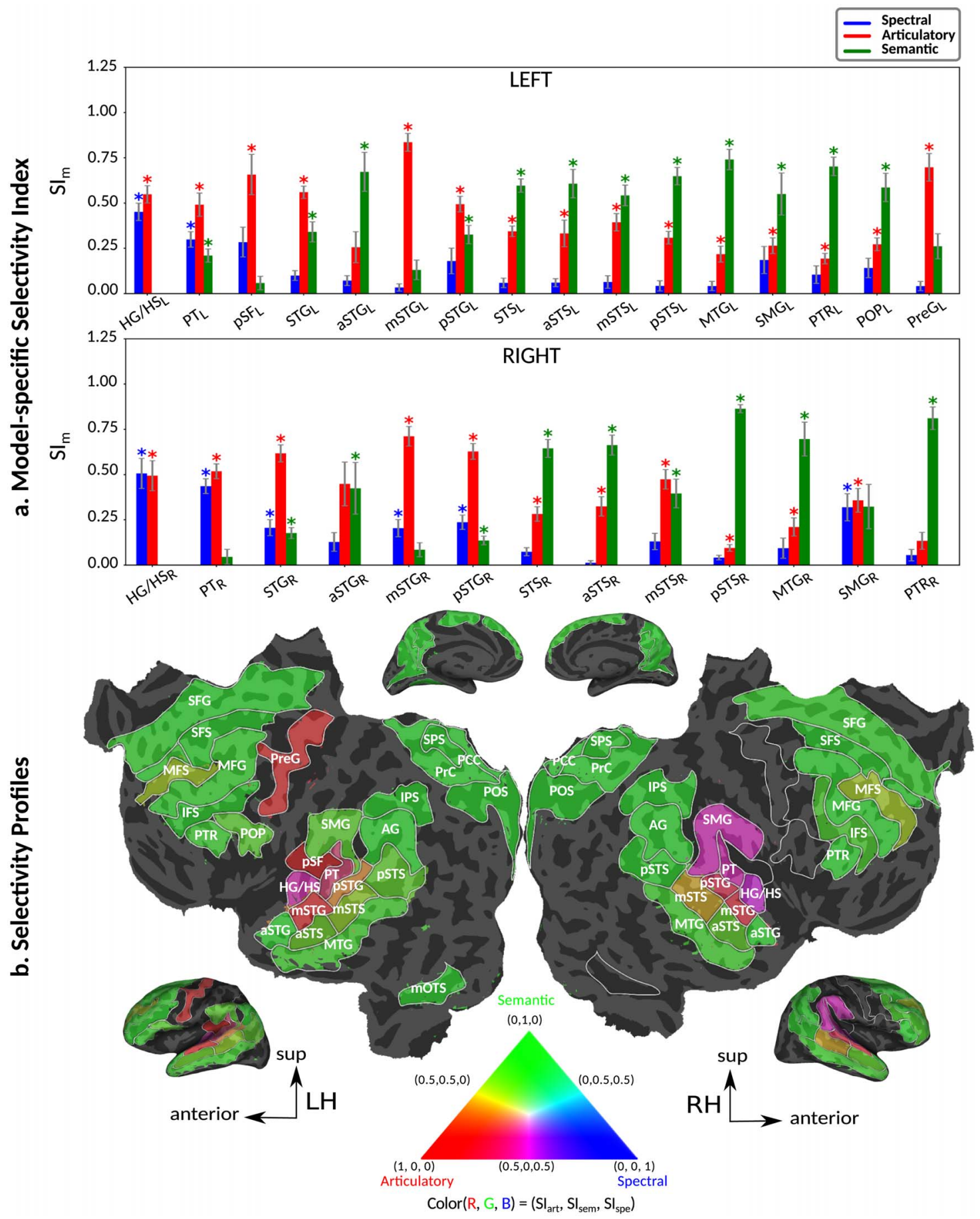

Figure 4. Selectivity for multilevel speech features. (a) "Model-specific selectivity indices." Single-voxel prediction scores on passive-listening data were used to quantify the selectivity of each ROI to underlying model features. Model-specific prediction scores were averaged across speech-selective voxels within each ROI and normalized such that the cumulative score from all models was 1 . The resultant measure was taken as a model-specific selectivity index, $\left(\mathrm{SI}_{m}\right)$. $\mathrm{SI}_{m}$ is in the range of $[0,1]$, where higher values indicate stronger selectivity for the underlying model. Bar plots display SI $m$ for spectral, articulatory, and semantic models (mean \pm standard error of mean (SEM) across subjects). Significant indices are marked with $*(P<0.05$; see Supplementary Fig. 3a-e for selectivity indices of individual subjects). ROIs in perisylvian cortex are displayed (see Supplementary Fig. 2 for nonperisylvian ROIs; see Materials and Methods for ROI abbreviations). ROIs in LH and RH are shown in the top and bottom panels, respectively. $\mathrm{POP}_{\mathrm{R}}$ and $\mathrm{PreG}_{\mathrm{R}}$ that did not have consistent speech selectivity in individual subjects were excluded (see Materials and Methods). (b) "Intrinsic selectivity profiles." Selectivity profiles of cortical ROIs averaged across subjects are shown on the cortical flatmap of a representative subject (S4). Significant articulatory, semantic, and spectral selectivity indices of each ROI are projected to the red, green, and blue channels of the RGB colormap (see Materials and Methods). This analysis only included ROIs with consistent selectivity for speech features in each individual subject. Medial and lateral views of the inflated hemispheres are also shown. A progression from low-intermediate to high-level speech representations are apparent across bilateral temporal cortex in the superior-inferior direction; consistently in all subjects (see Supplementary Fig. 4 for selectivity profiles of individual subjects). Meanwhile, semantic selectivity is dominant in many higher-order 
2017). Supplementary Fig. 1 displays the cortical distribution of prediction scores for each model in a representative subject, and Supplementary Table 2 lists the number of significantly predicted voxels by each model in anatomical ROIs. We find "spectrally selective voxels" mainly in early auditory regions (bilateral HG/HS and PT; and left pSF) and bilateral SMG, and "articulatorily selective voxels" mainly in early auditory regions (bilateral HG/HS and PT; and left pSF), bilateral STG, STS, SMG, and MFS as well as left POP and PreG. In contrast, "semantically selective voxels" are found broadly across cortex except early auditory regions (bilateral HG/HS and right PT).

To quantitatively examine cortical overlap among spectral, articulatory, and semantic representations, we separately measured the degree of functional selectivity for each feature level via a model-specific selectivity index $\left(\mathrm{SI}_{m}\right.$; see Materials and Methods). Bar plots of selectivity indices are displayed in Figure $4 a$ for perisylvian cortex and in Supplementary Fig. 2 for nonperisylvian cortex (see Supplementary Fig. 3a-e for singlesubject results). Distinct selectivity profiles are observed from distributed selectivity for spectral, articulatory, and semantic features (e.g., left PT and right pSTG) to strong tuning to a single level of features (e.g., left IPS and PCC). The selectivity profiles of the ROIs are also visualized on the cortical flatmap projecting articulatory, semantic, and spectral selectivity indices of each ROI to the red, green, and blue channels of the RGB colormap as seen in Figure $4 b$ (see Supplementary Fig. 4 for selectivity profile flatmaps in individual subjects; see Materials and Methods for colormap details). A progression from lowintermediate to high-level speech representations is apparent across bilateral temporal cortex in superior-inferior direction (HG/HS $\rightarrow$ mSTG $\rightarrow$ mSTS $\rightarrow$ MTG) consistently in all subjects. Furthermore, many higher-order regions in parietal (bilateral AG, IPS, SPS, PrC, PCC, and POS) and frontal cortices (bilateral PTR, IFS, MFG, SFS, and SFG; and left POP) manifest dominant semantic selectivity consistently in all subjects $(P<0.05$; see Supplementary Fig. $3 a-e$ for single-subject results). To examine the hierarchical organization of the speech representations in a finer scale, we also defined a complexity index, CI, that reflects whether an ROI is relatively tuned for low-level spectral or high-level semantic features. A detailed investigation of the gradients in CI across 2 main auditory streams (dorsal and ventral stream) was conducted (see Supplementary Results). These results corroborate the view that speech representations are hierarchically organized across cortex with partial overlap mostly in early and intermediate stages of speech processing.

Next, we systematically examined attentional modulations at each level of speech representation during a diotic cocktailparty task. To do this, we recorded whole-brain BOLD responses while participants listened to temporally overlaid spoken narratives from 2 different speakers and attended to either a male or female speaker in these 2-speaker stories. We used the spectral, articulatory, and semantic models fit using passive-listening data to predict responses during the cocktail-party task. Since a voxel can represent information on both attended and unattended stimuli, response predictions were expressed as a convex combination of individual predictions for the attended and unattended story within each 2-speaker story. Prediction scores were computed based on estimated responses as the combination weights were varied in [0 1] (see Materials and Methods). Scores for the optimal combination model were compared against the scores from the individual models for attended and unattended stories. If the optimal combination model significantly outperforms the individual models, it indicates that the voxel represents information from both attended and unattended stimuli.

Figure 5 displays prediction scores of the spectral, articulatory, and semantic models as a function of the combination weight in representative ROIs, including HG, HS, and PT. Scores based on only attended story $\left(r_{a}\right)$, based on only the unattended story $\left(r_{u}\right)$, and based on the optimal combination of the two $\left(r_{\max }\right)$ are marked. A diverse set of attentional effects are observed for each type of model. For the "spectral model" in left HG/HS, the optimal combination assigns matched weights to attended and unattended stories, and $r_{\text {max }}$ is larger than $r_{a}\left(P<10^{-4}\right)$. This finding implies that spectral representations of the unattended story are mostly maintained; and there is no apparent bias toward the attended story at spectral level in left HG/HS. For the "articulatory model" in left HG/HS, $r_{a}$ is larger than $r_{u}\left(P<10^{-4}\right)$, whereas $r_{\max }$ is greater than $r_{a}\left(P<10^{-2}\right)$. Besides, the optimal combination gives slightly higher weight to the attended versus unattended story. This result suggests that attention moderately shifts articulatory representations in left HG/HS in favor of the attended stream such that articulatory representations of the unattended story are preserved to a degree. For the "semantic model" in left PT, $r_{a}$ is much higher than $r_{u}\left(P<10^{-4}\right)$. Besides, the optimal combination assigns substantially higher weight to the attended story in this case. This finding indicates that attention strongly shifts semantic representations in left PT toward the attended stimulus. A simple inspection of these results suggests that attention may have distinct effects at various levels of speech representation across cortex. Hence, a detailed quantitative analysis is warranted to measure the effect of attention at each level.

\section{Level-Specific Attentional Modulations}

To quantitatively assess the strength and direction of attentional modulations, we separately investigated the modulatory effects on spectral, articulatory, and semantic features across cortex. To measure modulatory effects at each feature level, a model-specific attention index $\left(\mathrm{AI}_{m}\right)$ was computed, reflecting the difference in model prediction scores when the stories were attended versus unattended (see Materials and Methods). $\mathrm{AI}_{m}$ is in the range of $[-1,1]$; a positive index indicates selectivity modulation in favor of the attended stimulus, whereas a negative index indicates selectivity modulation in favor of the unattended stimulus. A value of zero indicates no modulation.

Figure $6 a$ and Supplementary Figure 7 display the attention index for spectral, articulatory, and semantic models across perisylvian and nonperislyvian ROIs, respectively (see Supplementary Fig. 8a-e for single-subject results). The modulation profiles of the ROIs are also visualized on the cortical flatmap, projecting articulatory, semantic, and spectral attention indices to the red, green, and blue channels of the RGB colormap as seen in Figure $6 b$ (see Supplementary Fig. 9 


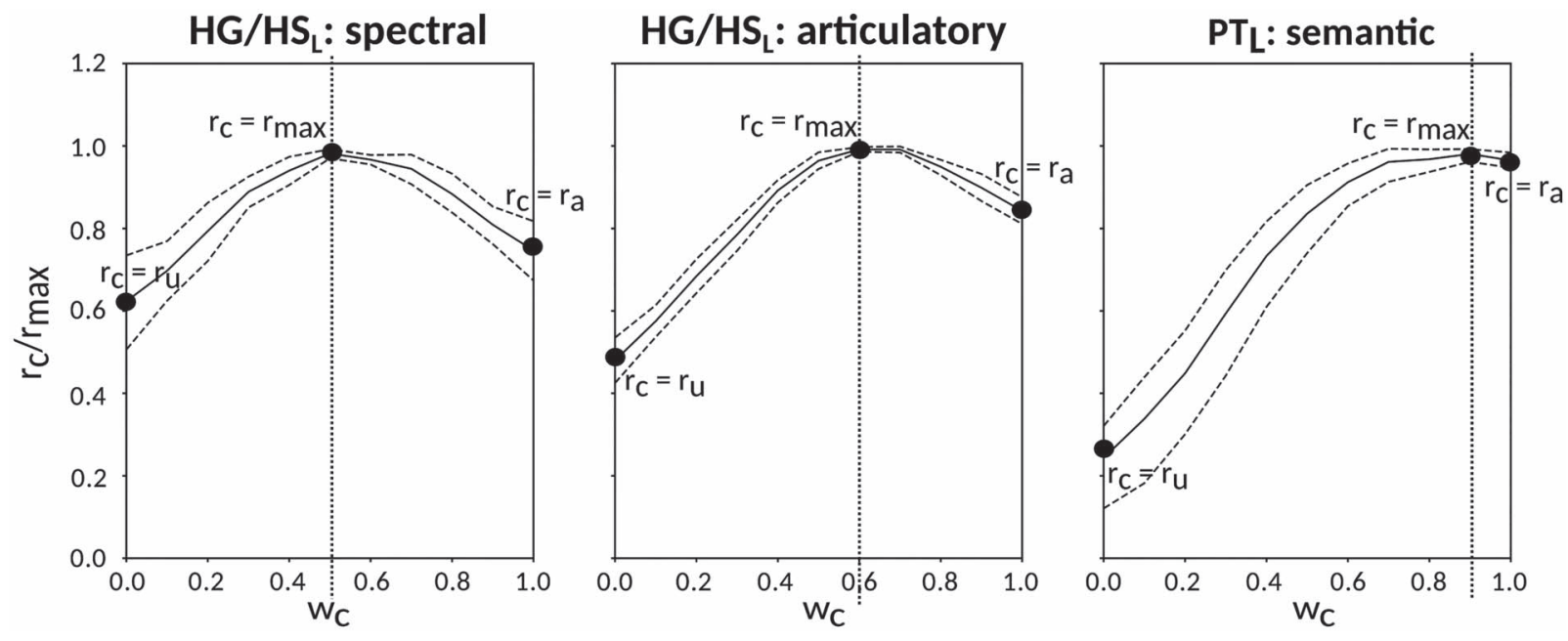

Figure 5. Predicting cocktail-party responses. Passive-listening models were tested during the cocktail-party task by predicting BOLD responses in the cocktail-party data. Since a voxel might represent information from both attended and unattended stimuli, response predictions were expressed as a convex combination of individual predictions for the attended and unattended story within each 2-speaker story. Prediction scores were computed as the combination weights $\left(w_{c}\right)$ were varied in [0 1 ] (see Materials and Methods). Prediction scores for a given model were averaged across speech-selective voxels within each ROI $\left(r_{c}\right)$. The normalized scores of spectral, articulatory, and semantic models are displayed in several representative ROIs (HG/HS, HG/HS, and PT). Solid and dashed lines indicate mean and 95\% confidence intervals across subjects. Scores based on only the attended story $\left(r_{a}\right)$, based on only the unattended story $\left(r_{u}\right)$, and based on the optimal combination of the two $\left(r_{\text {max }}\right)$ are marked with circles. For the "spectral model" in left HG/HS, $r_{\max }$ is larger than $r_{a}\left(\mathrm{P}<10^{-4}\right)$; and the optimal combination equally weighs attended and unattended stories. For the "articulatory model" in left HG/HS, $r_{a}$ is larger than $r_{u}\left(P<10^{-4}\right)$, whereas $r_{\text {max }}$ is greater than $r_{a}\left(P<10^{-2}\right)$. Besides, the optimal combination puts slightly higher weight to attended story than unattended story. For the "semantic model" in left PT, $r_{a}$ is much higher than $r_{u}\left(P<10^{-4}\right)$, and the optimal combination puts much greater weight to attended story than unattended one. These representative results imply that attention may have divergent effects at various levels of speech representations across cortex.

for modulation profile flatmaps in individual subjects). Here we discuss the attention index for each model individually. "Spectral modulation" is not consistently significant in each subject across perisylvian ROIs $(P>0.05)$. On the other hand, moderate spectral modulation is found in right SFG consistently in all subjects $\left(P<10^{-3}\right)$. "Articulatory modulation" starts as early as HG/HS bilaterally $\left(P<10^{-3}\right)$. In the dorsal stream, it extends to PreG and POP in the left hemisphere (LH) and to SMG in the right hemisphere (RH; $P<10^{-2}$ ); and it becomes dominant only in left PreG consistently in all subjects $(P<0.05)$. In the ventral stream, it extends to left PTR and bilateral MTG $\left(P<10^{-2}\right)$. Articulatory modulation is also found-albeit generally less strongly-in frontal regions (bilateral MFS; left MFG; and right IFS and SFG) consistently in all subjects $(P<0.05)$. In the dorsal stream, "semantic modulation" starts in PT and extends to POP in $\mathrm{LH}\left(\mathrm{P}<10^{-2}\right)$, whereas it is not apparent in the right dorsal stream $(P>0.05)$. In the ventral stream, semantic modulation starts in aSTG and mSTS bilaterally $(P<0.05)$. It extends to MTG and PTR, and becomes dominant in both ends of the bilateral ventral stream $(P<0.05)$. Lastly, semantic modulation is observed widespread across higher-order regions within frontal and parietal cortices consistently in all subjects $(P<0.05)$, with the exception of left IPS $(P>0.05)$. Taken together, these results suggest that attending to a target speaker alters articulatory and semantic representations broadly across cortex.

\section{Global Attentional Modulations}

It is commonly assumed that attentional effects grow stronger toward higher-order regions across the cortical hierarchy of speech (Zion Golumbic et al. 2013; O'Sullivan et al. 2019; Regev et al. 2019). Yet, a systematic examination of attentional modulation gradients across dorsal and ventral streams is lacking. To examine this issue, we measured overall attentional modulation in each region via a gAI (see Materials and Methods). Similar to the model-specific attention indices, a positive gAI indicates modulations in favor of the attended stimulus, and a negative gAI indicates modulations in favor of the unattended stimulus.

1. Dorsal stream. We first examined variation of gAI across the dorsal stream (left dorsal-1: $\mathrm{HG} / \mathrm{HS}_{\mathrm{L}} \rightarrow \mathrm{PT}_{\mathrm{L}} \rightarrow\left(\mathrm{SMG}_{\mathrm{L}}\right)$ $\rightarrow \mathrm{POP}_{\mathrm{L}}$, left dorsal-2: $\mathrm{HG} / \mathrm{HS}_{\mathrm{L}} \rightarrow \mathrm{PT}_{\mathrm{L}} \rightarrow\left(\mathrm{SMG}_{\mathrm{L}}\right) \rightarrow \mathrm{PreG}_{\mathrm{L}}$, and right dorsal: $\mathrm{HG} / \mathrm{HS}_{\mathrm{R}} \rightarrow \mathrm{PT}_{\mathrm{R}} \rightarrow \mathrm{SMG}_{\mathrm{R}}$ ) as shown in Figure 7. We find significant increase in gAI across the following left dorsal subtrajectories consistently in all subjects $(P<0.05$; see Supplementary Figure 11 for gradients in individual subjects): gAI $_{\mathrm{PT}}<\mathrm{gAI}_{\mathrm{SMG}}<\mathrm{gAI}_{\mathrm{POP}}$ and $\mathrm{gAI}_{\mathrm{PT}}<$ $\mathrm{gAI}_{\mathrm{SMG}}<\mathrm{gAI}_{\text {PreG }}$. In contrast, we find no consistent gradient in the right dorsal stream $(P>0.05)$. These results suggest that attentional modulations grow progressively stronger across the dorsal stream in LH.

2. Ventral stream. We then examined variation of gAI across the ventral stream (left ventral-1: HG/HS $\rightarrow$ mSTG $_{\mathrm{L}} \rightarrow$ $\mathrm{mSTS}_{\mathrm{L}} \rightarrow \mathrm{MTG}_{\mathrm{L}}$, left ventral-2: $\mathrm{HG} / \mathrm{HS}_{\mathrm{L}} \rightarrow \mathrm{mSTG}_{\mathrm{L}} \rightarrow \mathrm{aSTG}_{\mathrm{L}}$ $\rightarrow \mathrm{PTR}_{\mathrm{L}}$, right ventral-1: $\mathrm{HG} / \mathrm{HS}_{\mathrm{R}} \rightarrow \mathrm{mSTG}_{\mathrm{R}} \rightarrow \mathrm{mSTS}_{\mathrm{R}} \rightarrow$ $\mathrm{MTG}_{\mathrm{R}}$ and right ventral-2: $\mathrm{HG} / \mathrm{HS}_{\mathrm{R}} \rightarrow \mathrm{mSTG}_{\mathrm{R}} \rightarrow \mathrm{aSTG}_{\mathrm{R}} \rightarrow$ $\mathrm{PTR}_{R}$ ), as shown in Figure 7. We find significant increase in gAI across the following subtrajectories consistently in all subjects $(P<0.05$; see Supplementary Fig. 11 for gradients in individual subjects): $\mathrm{gAI}_{\mathrm{HG} / \mathrm{HS}}<\mathrm{gAI}_{\mathrm{mSTG}}<\mathrm{gAI}_{\mathrm{aSTG}}$ and $\mathrm{gAI}_{\mathrm{HG} / \mathrm{HS}}<\mathrm{gAI}_{\mathrm{mSTG}}<\mathrm{gAI}_{\mathrm{mSTS}}$ in the left ventral stream, and $\mathrm{gAI}_{\mathrm{mSTG}}<\mathrm{gAI}_{\mathrm{aSTG}}$ in the right ventral stream. In contrast, we find no difference between aSTG and PTR bilaterally, between mSTS and MTG in the left ventral stream, and between HG/HS, mSTG, mSTS, and MTG in the right ventral stream $(P>0.05)$. These results suggest that overall attentional 


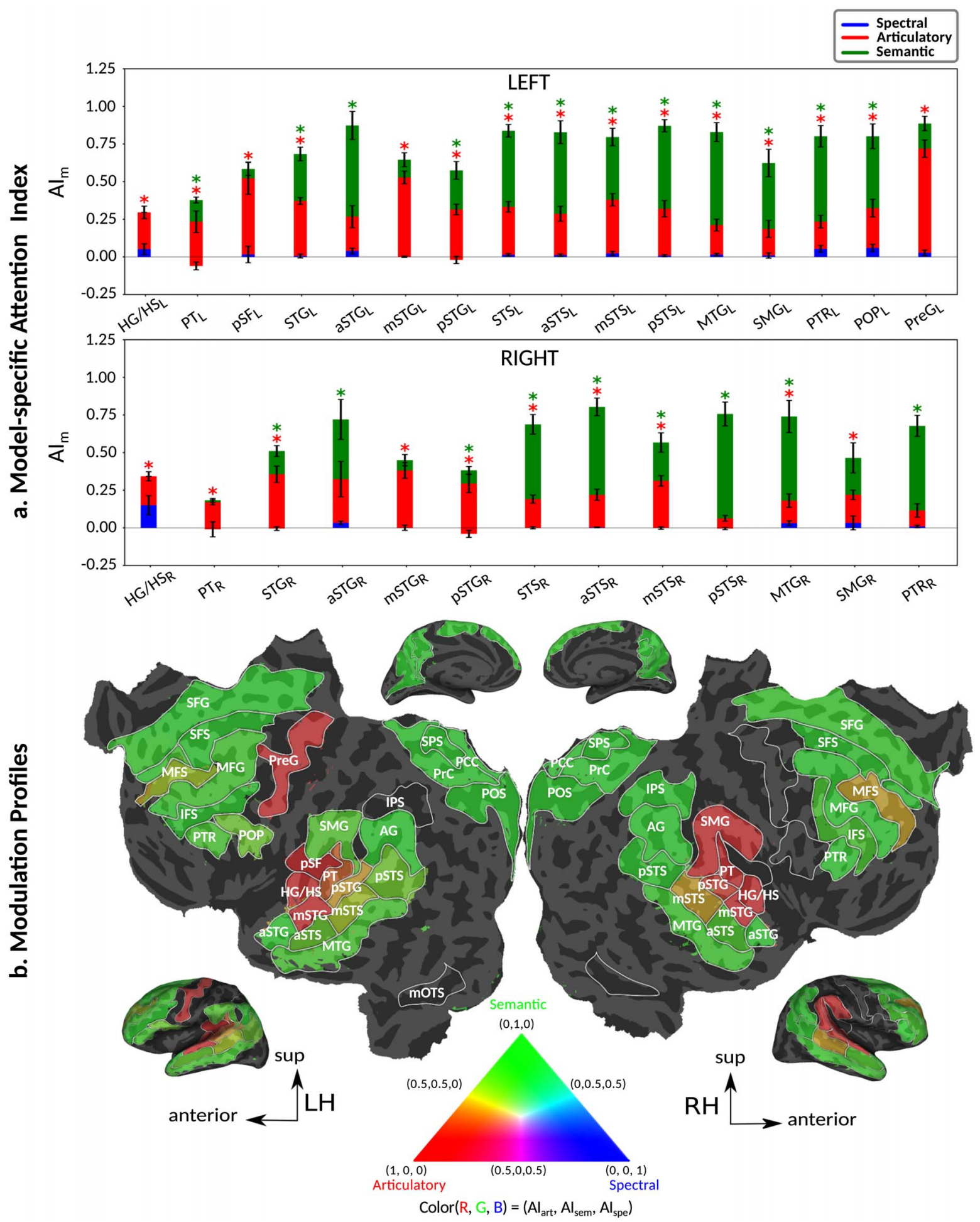

Figure 6. Attentional modulation of multilevel speech representations. (a) "Model-specific attention indices." A model-specific attention index (AI $m$ ) was computed based on the difference in model prediction scores when the stories were attended versus unattended (see Materials and Methods). $\mathrm{AI}_{m}$ is in the range of [-1,1], where a positive index indicates modulation in favor of the attended stimulus and a negative index indicates modulation in favor of the unattended stimulus. For each ROI in perisylvian cortex, spectral, articulatory, and semantic attention indices are given (mean \pm SEM across subjects), and their sum yields the overall modulation (see Supplementary Fig. 7 for nonperisylvian ROIs). Significantly positive indices are marked with $*(P<0.05$, bootstrap test; see Supplementary Fig. 8a-e for attention indices of individual subjects). ROIs in the LH and RH are shown in top and bottom panels, respectively. These results show that selectivity modulations distribute broadly across cortex at the linguistic level (articulatory and semantic). (b) "Attentional modulation profiles." Modulation profiles averaged across subjects are displayed on the flattened cortical surface of a representative subject (S4). Significantly positive articulatory, semantic, and spectral attention indices are projected onto the 
modulations gradually increase across the ventral stream, and that the increases are more consistent in LH compared with $\mathrm{RH}$.

3. Representational complexity versus attentional modulation. Visual inspection of Supplementary Figure $6 b$ and Figure $7 b$ suggests that the subtrajectories with significant increases in CI and in gAI overlap largely in left ventral stream and partly in left dorsal stream. To quantitatively examine the overlap in left ventral stream, we analyzed the correlation between $\mathrm{CI}$ and gAI across the left-ventral subtrajectories where significant increases in CI are observed. We find significant correlations in $\mathrm{HG} / \mathrm{HS} \rightarrow \mathrm{mSTG} \rightarrow \mathrm{mSTS}$ and in HG/HS $\rightarrow$ mSTG $\rightarrow$ aSTG $\left(r>0.98\right.$, bootstrap test, $\left.P<10^{-4}\right)$ consistently in all subjects. In line with a recent study arguing for stronger attentional modulation and higher representational complexity in STG compared with HG (O'Sullivan et al. 2019), our results indicate that attentional modulation increases toward higher-order regions as the representational complexity increases across the dorsal and ventral streams in LH (more apparent in ventral than dorsal stream).

4. Hemispheric asymmetries in attentional modulation. To assess potential hemispheric asymmetries in attentional modulation, we compared gAI between the left and right hemispheric counterparts of each ROI. This analysis was restricted to ROIs with consistent selectivity for speech features in both hemispheres in each individual subject (see Materials and Methods). Supplementary Table 3 lists the results of the across-hemisphere comparison. No consistent hemispheric asymmetry is found across cortex with the exception of mSTG having a left-hemispheric bias in gAI consistently in all subjects $(P<0.05)$. These results indicate that there is mild lateralization in attentional modulation of intermediate-level speech features.

\section{Cortical Representation of Unattended Speech}

An important question regarding multispeaker speech perception is to what extent unattended stimuli are represented in cortex. To address this question, here we investigated spectral, articulatory, and semantic representations of unattended stories during the cocktail-party task. We reasoned that if significant information about unattended speech is represented in a brain region, then features of unattended speech should explain significant variance in measured BOLD responses. To test this, we compared the prediction score of a combination model comprising the features of both attended and unattended stories (optimal convex combination) against the prediction score of an individual model comprising only the features of the attended story (see Materials and Methods). If the combination model significantly outperforms the individual model in an ROI, then the corresponding features of unattended speech are significantly represented in that ROI.

Figure 8 displays model performance when responses are predicted based on speech features from the attended story alone, and when they are instead predicted based on the optimally combined features from the attended and unattended stories. Results are shown for each ROI along the dorsal and ventral streams and in the left and right hemispheres (see Supplementary Fig. 12a-e for single-subject results). Along the left (HG/HS $\rightarrow$ PT $\rightarrow$ SMG $\rightarrow$ (POP, PreG)) and right (HG/HS $\rightarrow$ $\mathrm{PT} \rightarrow \mathrm{SMG}$ ) dorsal stream, spectral features of unattended speech are represented up to PT in LH and up to SMG in RH $(P<0.01)$, articulatory features are represented bilaterally up to PT $(P<0.05)$, whereas no semantic representation is apparent $(P>0.05)$. Along the left ventral stream (HG/HS $\rightarrow$ mSTG $\rightarrow$ mSTS $\rightarrow$ MTG and HG/HS $\rightarrow$ mSTG $\rightarrow$ aSTG $\rightarrow$ PTR), spectral and articulatory features are represented in HG/HS $\left(P<10^{-4}\right)$, again with no semantic representation $(P>0.05)$. In the right ventral stream $(\mathrm{HG} / \mathrm{HS} \rightarrow \mathrm{mSTG} \rightarrow \mathrm{mSTS} \rightarrow$ MTG and HG/HS $\rightarrow$ $\mathrm{mSTG} \rightarrow$ aSTG $\rightarrow$ PTR), spectral features are represented in $\mathrm{HG} / \mathrm{HS}$; articulatory features are represented up to mSTG $(P<0.05)$; and semantic features are represented only in mSTS $(P<0.05)$. These results indicate that cortical representations of unattended speech in multispeaker environments extend from the spectral to the semantic level, albeit semantic representations are constrained to right parabelt auditory cortex (mSTS). Furthermore, representations of unattended speech are more broadly spread across the right hemisphere. Note that prior studies have reported response correlations and anatomical overlap between these belt/parabelt auditory regions and the reorienting attention system in the right-hemisphere (Corbetta et al. 2008; Vossel et al. 2014; Puschmann et al. 2017). Therefore, relatively broader representations of unattended speech in the right hemisphere might facilitate distractor detection and filtering during auditory attention tasks.

\section{Discussion}

In this study, we investigated the effects of auditory attention on multilevel speech representations across cortex during a diotic cocktail-party task with naturalistic stimuli composed of spoken narratives. To assess baseline selectivity for multilevel speech features, we first fit spectral, articulatory, and semantic models using responses recorded during passive listening. We then quantified the complexity of intrinsic representations in each brain region. Next, we used fit models that reflect baseline selectivity for speech features to assess attentional modulation of speech representations. To do this, responses predicted using stimulus features of attended and unattended stories were compared with responses recorded during the cocktail-party task. This study is among the first to quantitatively characterize attentional modulations in multilevel speech representations of attended and unattended stimuli across speech-related cortex.

\section{Attentional Modulations}

The effects of auditory attention on cortical responses have been primarily examined in the literature using controlled stimuli such as simple tones, melodies, and isolated syllables or words (Alho et al. 1999; Jäncke et al. 2001, 2003; Lipschutz et al. 2002;

red, green and blue channels of the colormap (see Materials and Methods). A progression in the level of speech representations dominantly modulated is apparent from HG/HS to MTG across bilateral temporal cortex (see Supplementary Fig. 9 for modulation profiles of individual subjects). Articulatory modulation is dominant in one end of the dorsal stream (left PreG), whereas semantic modulation becomes dominant in both ends of the ventral stream (bilateral PTR and MTG) ( $<<0.05$; see Supplementary Figs. $8 a-e$ and 9). On the other hand, semantic modulation is dominant in most of the higher-order regions in the parietal and frontal cortices consistently in all subjects (bilateral AG, SPS, PrC, PCC, POS, SFG, SFS, and PTR; left MFG; and right IPS) (P<0.05; see Supplementary Fig. 8a-e). 


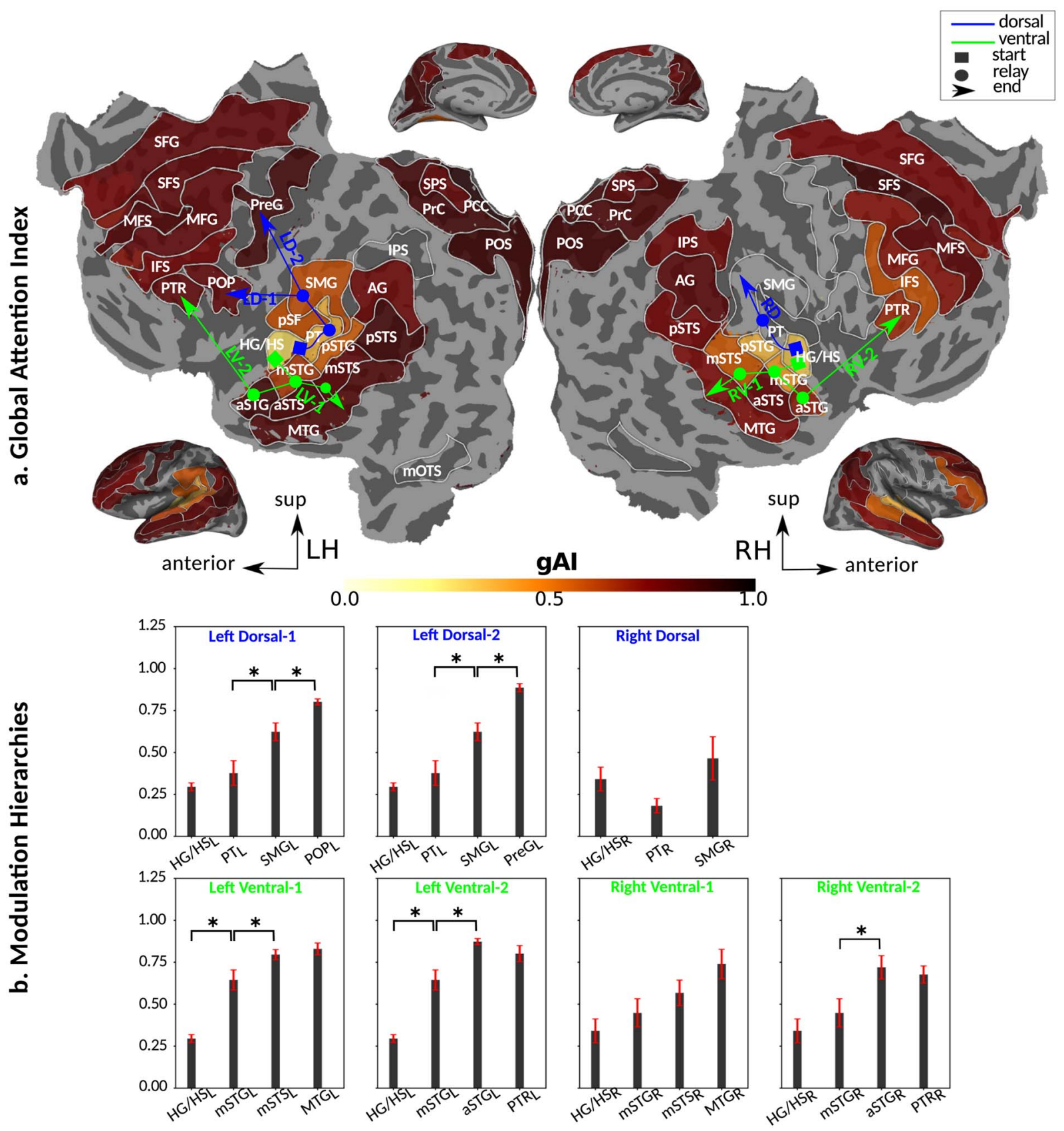

Figure 7. Global attentional modulation. (a) "Global attention index." To quantify overall modulatory effects on selectivity across all examined feature levels, global attentional modulation (gAI) was computed by summing spectral, articulatory, and semantic attention indices (see Materials and Methods). gAI is in the range of [-1,1] and a value of zero indicates no modulation. Colors indicate significantly positive gAI averaged across subjects (see legend; see Supplementary Fig. 10 for bar plots of gAI across cortex). Dorsal and ventral pathways are shown with blue and green lines, respectively: left dorsal-1 (LD-1), left dorsal-2 (LD-2) and right dorsal (RD), left ventral-1 (LV-1), left ventral-2 (LV-2), right ventral-1 (RV-1) and right ventral-2 (RV-2). Squares mark regions where pathways begin; arrows mark regions where pathways end; and circles mark relay regions in between. (b) "Modulation hierarchies." Bar plots display gAI (mean \pm SEM across subjects) along LD-1, LD-2, RD, LV-1, LV-2, RV-1 and RV-2, shown in separate panels. Significant differences in gAI between consecutive ROIs are marked with brackets $(P<0.05$, bootstrap test; see Supplementary Fig. 11 for single-subject results). Significant gradients in gAI are gAI $I_{\mathrm{PT}}<\mathrm{gAI}_{\mathrm{SMG}}<\mathrm{gAI}_{\mathrm{POP}}$ in LD-1, gAI $\mathrm{PT}_{\mathrm{PT}}<\mathrm{gAI}_{\mathrm{SMG}}<\mathrm{gAI}_{\mathrm{PreG}}$ in LD-2, gAI $\mathrm{HG} / \mathrm{HS}<\mathrm{gAI}_{\mathrm{mSTG}}<\mathrm{gAI}_{\mathrm{mSTS}}$ in LV-1, $\mathrm{gAI}_{\mathrm{HG} / \mathrm{HS}}<\mathrm{gAI}_{\mathrm{mSTG}}<\mathrm{gAI}_{\mathrm{aSTG}}$ in LV-2, and $\mathrm{gAI}_{\mathrm{mSTG}}<\mathrm{gAI}_{\mathrm{aSTG}}$ in RV-2. In the LH, gAI gradually increases from early auditory regions to higher-order regions across the dorsal and ventral pathways. Similar patterns are also observed in the right hemisphere, although the gradients in gAI are less consistent across subjects.

Petkov et al. 2004; Johnson and Zatorre 2005; Degerman et al. 2006; Rinne et al. 2005, 2008; Rinne 2010; Woods et al. 2009, 2010; Paltoglou et al. 2009; Da costa et al. 2013; Seydell-Greenwald et al. 2014; Riecke et al. 2017). As such, less is known regarding how attention alters hierarchical representations of natural speech.
Recent studies on this topic have mainly reported attentional modulations of low-level speech representations comprising speech-envelope and spectrogram features in early auditory and higher-order regions during the cocktail-party task (Mesgarani and Chang 2012; Ding and Simon 2012a, 2012b; Zion Golumbic 

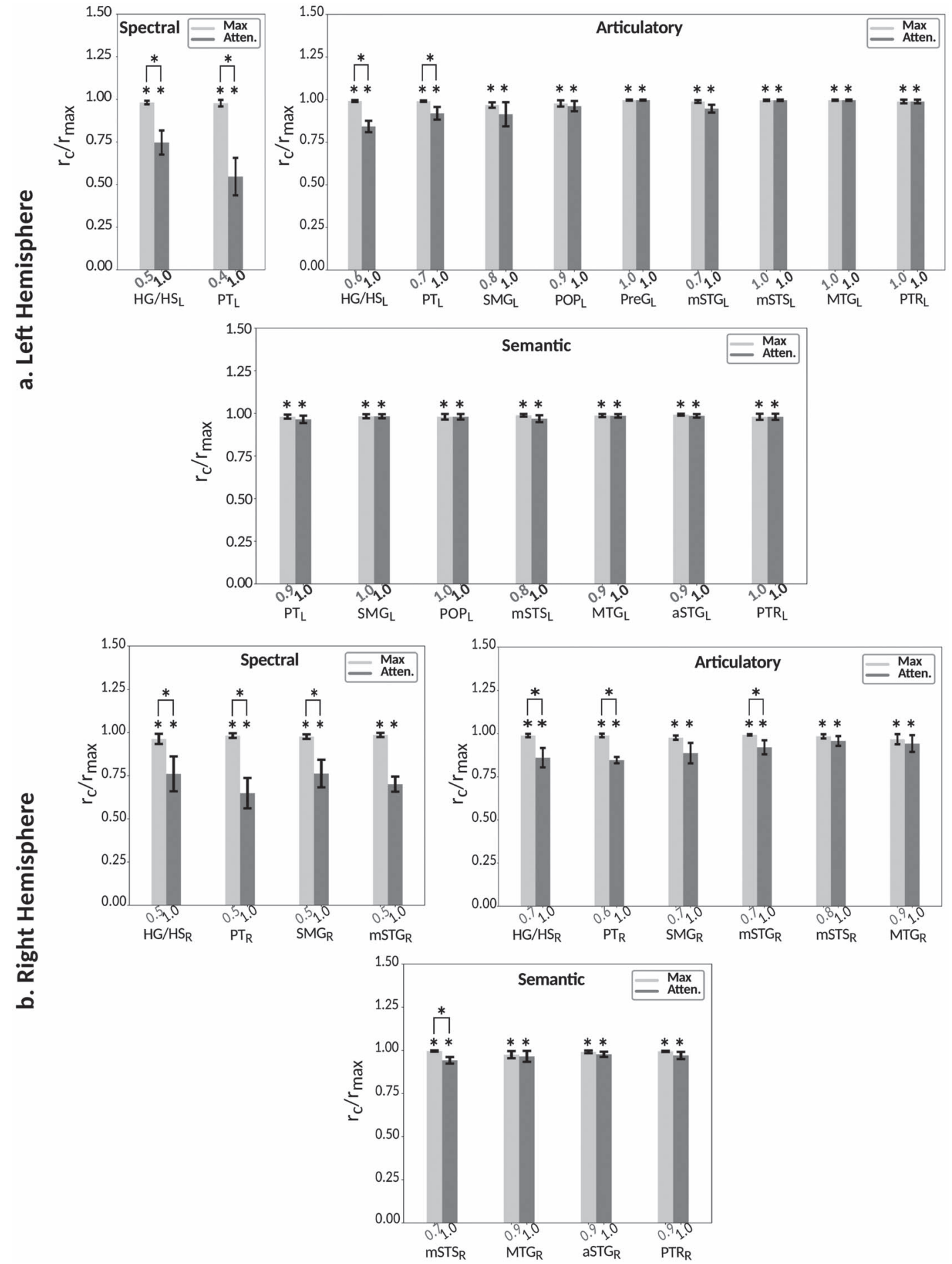

Figure 8. Representation of unattended speech. Passive-listening models were tested on cocktail-party data to assess representation of unattended speech during the cocktail-party task. Prediction scores were calculated separately for a combination model comprising features of both attended and unattended stories $\left(r_{\text {max }}\right.$ : optimal convex combination) and an individual model only comprising features of the attended story $\left(r_{a}\right)$. Significant difference in prediction between the 2 models is an indication that BOLD responses carry significant information on unattended speech. Bar plots display normalized prediction scores (mean \pm SEM across subjects; combination model in light gray and individual model in gray). Significant scores are marked with $*\left(P<10^{-4}\right.$, bootstrap test; see Supplementary Fig. $12 a-e$ for single-subject results), and significant differences are marked with brackets $(P<0.05)$. Prediction scores are displayed for ROIs in the dorsal and ventral streams, with significant selectivity for given model features. (a) "LH." "Spectral representations" of unattended speech extend up to PT across the dorsal stream $(\mathrm{HG} / \mathrm{HS} \rightarrow \mathrm{PT} \rightarrow \mathrm{SMG} \rightarrow$ (POP, PreG) $)$ and are constrained to HG/HS across the ventral stream (HG/HS $\rightarrow \mathrm{mSTG} \rightarrow \mathrm{mSTS} \rightarrow \mathrm{MTG}$ and HG/HS $\rightarrow$ mSTG $\rightarrow$ aSTG $\rightarrow$ PTR) "Articulatory representations" of unattended speech extend up to PT across the dorsal stream and are constrained to HG/HS across the ventral stream. No "significant 
et al. 2013; Puvvada and Simon 2017; Puschmann et al. 2019). Going beyond, here we have explored attentional modulations spanning from low-level spectral to high-level semantic features. Although our results indicate that attentional modulations for articulatory and semantic representations distribute broadly across cortex, we find no consistent modulations for spectral representations in speech-related regions. Note that speech envelope and spectrogram features in natural speech carry intrinsic information about linguistic features including syllabic boundaries and articulatory features (Ding and Simon 2014; Di Liberto et al. 2015). These stimulus correlations can render it challenging to dissociate unique selectivity for articulatory versus spectral features. To minimize biases from potential stimulus correlations, here we leveraged a decorrelation procedure to obtain orthogonal spectral, articulatory, and semantic feature matrices for the stimulus. Therefore, the distinct modeling procedures for natural speech features might have contributed to the disparities between the current and previous studies on the existence of spectral modulations.

An important question regarding auditory attention is how the strength of attentional effects is distributed across cortex. A common view is that attentional modulations grow relatively stronger toward later stages of processing (Zion Golumbic et al. 2013). Recent studies support this view by reporting bilaterally stronger modulations in frontal versus temporal cortex (Regev et al. 2019) and in nonprimary versus primary auditory cortex (O'Sullivan et al. 2019). Adding to this body of evidence, we further show that attentional modulations gradually increase across the dorsal and ventral streams in the LH, as the complexity of speech representations grow. Although a similar trend is observed across the right hemisphere, gradients in attentional modulation are less consistent in right belt and parabelt auditory regions including PT. Furthermore, attentional modulations are weaker in the right versus LH within these regions. Note that belt and parabelt regions are suggested to be connected to the right temporoparietal junction (TPJ) during selective listening (Puschmann et al. 2017). TPJ is one of the central nodes in the reorienting attention system that monitors salient events to filter out distractors and help maintaining focused attention (Corbetta and Shulman 2002; Corbetta et al. 2008; Vossel et al. 2014). Hence, less consistent gradients and relatively weaker attentional modulations in right belt and parabelt auditory regions might suggest a functional role for these regions in detecting salient events within the unattended stream during selective listening tasks.

Another central question regarding mechanisms of selective attention in a multispeaker environment is how attentional modulations distribute across well-known dorsal and ventral streams. The dorsal stream that hosts articulatory representations is commonly considered to be involved in soundto-articulation mapping (Hickok and Poeppel 2007, 2016; Rauschecker and Scott 2009; Friederici 2011; Rauschecker 2011). The motor-theory of speech perception suggests that dorsal articulatory representations carry information about articulatory gestures of the speaker to facilitate the listener's comprehension (Liberman and Mattingly 1985; Hickok and Poeppel 2004; Davis and Johnsrude 2007; Scott et al. 2009b;
Möttönen et al. 2013). Recent studies support this account by reporting enhanced activity in precentral gyrus and premotor cortex during challenging listening conditions (Osnes et al. 2011; Hervais-Adelman et al. 2012; Wild et al. 2012). In accordance with the motor theory of speech perception, here we find predominant articulatory selectivity and modulation due to selective listening in one end of the dorsal stream (PreG). These articulatory modulations might serve to increase sensitivity to the target speaker's gestures to facilitate speech comprehension during difficult cocktail-party tasks (Wild et al. 2012).

In contrast to the dorsal stream, the ventral stream has been implicated in sound-to-meaning mapping (Hickok and Poeppel 2007, 2016; Rauschecker and Scott 2009; Friederici 2011; Rauschecker 2011). Compatible with this functional role, the ventral stream is suggested to transform acoustic representations of linguistic stimuli into object-based representations (Bizley and Cohen 2013). Here, we find that representational complexity of the speech features gradually increases across bilateral ventral stream, and semantic representations become dominant at the ends of it (bilateral PTR and MTG). In addition, speech level of attentional modulation also progresses across the ventral stream, and strong and predominant semantic modulations manifest toward later stages. Hence, the ventral stream might serve as a stage for interplay between bottom-up processing and top-down attentional modulation to gradually form auditory objects during selective listening (Bizley and Cohen 2013; Shinn-Cunningham et al. 2017; Rutten et al. 2019).

\section{Representation of the Unattended Speech}

Whether unattended speech is represented in cortex during selective listening and if so, at what feature levels its representations are maintained are crucial aspects of auditory attention. Behavioral accounts suggest that unattended speech is primarily represented at the acoustic level (Cherry 1953; Broadbent 1958). Corroborating these accounts, recent electrophysiology studies have identified acoustic representations of unattended speech localized to auditory cortex (Ding and Simon 2012a, 2012b; Zion Golumbic et al. 2013; Puvvada and Simon 2017; Brodbeck et al. 2018b; O'Sullivan et al. 2019; Puschmann et al. 2019). In contrast, here we find that acoustic representations of unattended speech extend beyond the auditory cortex as far as SMG in the right dorsal stream. Because SMG partly overlaps with the reorienting attention system, unattended speech representations in this region might contribute to filtering of distractors during the cocktail-party task (Corbetta et al. 2008; Vossel et al. 2014).

A more controversial issue is whether unattended speech representations carry information at the linguistic level (Driver 2001; Lavie 2005; Boulenger et al. 2010; Bronkhorst 2015; Kidd and Colburn 2017). Prior studies on this issue are split between those suggesting the presence (Wild et al. 2012; Evans et al. 2016) versus absence (Sabri et al. 2008; Brodbeck et al. 2018b) of linguistic representations. Here, we find that articulatory representations of unattended speech extend up to belt/parabelt auditory areas in the bilateral dorsal stream and the right ventral stream. We further find semantic representation of unattended

semantic representation" is apparent. (b) "Right hemisphere." "Spectral representations" of unattended speech extend up to SMG across the dorsal stream and are constrained to HG/HS across the ventral stream. "Articulatory representations" of unattended speech extend up to PT across the dorsal stream, and up to mSTG across the ventral stream. "Semantic representations" are found only in mSTS. These results suggest that processing of unattended speech is not constrained at spectral level but extends to articulatory and semantic level. 
speech in the right ventral stream (mSTS). These linguistic representations of unattended speech are naturally weaker than those of attended speech, and they are localized to early-tointermediate stages of auditory processing. Our findings suggest that unattended speech is represented at the linguistic level prior to entering the broad semantic system where full selection of the attended stream occurs (Bregman 1994; Pulvermüller and Shtyrov 2006; Relander et al. 2009; Näätänen et al. 2011; Rämä et al. 2012; Bronkhorst 2015; Ding et al. 2018). Overall, these linguistic representations might serve to direct exogenous triggering of attention to salient features in unattended speech (Moray 1959; Treisman 1960, 1964; Wood and Cowan 1995; Driver 2001; Bronkhorst 2015). Meanwhile, attenuated semantic representations in the ventral stream might facilitate semantic priming of the attended stream by relevant information in the unattended stream (Lewis 1970; Driver 2001; Rivenez et al. 2006).

\section{Future Work}

Reliability of statistical assessments in neuroimaging depends on 2 main factors: sample size and amount of data collected per subject. Given experimental constraints, it is difficult to increase both factors in a single study. In this unavoidable trade-off, a common practice in fMRI studies is to collect a relatively limited dataset from more subjects. This practice prioritizes acrosssubject variability over within-subject variability, at the expense of individual-subject results. Diverting away from this practice, we collected a larger amount of data per subject to give greater focus to reliability in single subjects. This choice is motivated by the central aims of the voxelwise modeling (VM) approach. The VM framework aims to sensitively measure tuning profiles of single voxels in individual subjects. For the natural speech perception experiments conducted here, the tuning profiles were characterized over 3 separate high-dimensional spaces containing hundreds of acoustic and linguistic features. To maximize sensitivity of VM models, we conducted extensive experiments in each individual subject to increase the amount and diversity of fMRI data collected. This design enhanced the quality of resulting VM models and reliability of individual-subject results. Indeed, here we find highly uniform results across individual subjects, suggesting that the reported effects are highly robust. That said, across subject and across language variability might occur in diverse, multilingual cohorts. Assessment of attentional effects on speech representations in these broader populations remains important future work.

In the current study, subjects were presented continuous natural speech stimuli. In the passive-listening task, they were instructed to vigilantly listen to the presented story. Our analyses reveal that BOLD responses in large swaths of languagerelated areas can be significantly predicted by voxel-wise models comprising spectral, articulatory and semantic speech features. Moreover, the spectral, articulatory and semantic representations mapped in single subjects are highly consistent across subjects. Therefore, these results suggest that the participants performed reasonably well in active listening of singlespeaker stories. In the cocktail-party experiment, subjects were instead instructed to attentively listen to one of 2 speakers. Our analyses in this case reveal broad attentional modulations in representation of semantic information across cortex, in favor of the target speaker. Semantic features of natural speech show gradual variation across time compared with low-level spectral information. Therefore, this finding suggests that subjects also performed well during the sustained attention tasks in the cocktail-party task. That said, we cannot rule out momentary shifts in attention away from the target speaker. If momentary shifts toward the unattended speaker are frequent, they might increase the proportion of unattended speech information that BOLD responses carry. In turn, this might have contributed to the strength of unattended speech representations that we measured during the cocktail-party task. Postscan questionnaires that assess participants' comprehension of attended and unattended stories are a common control for task execution (Regev et al. 2019). However, postscan memory controls cannot guarantee the lack of momentary attention shifts that typically last less than $200 \mathrm{~ms}$ (Spence and Driver 1997). On the other hand, implementing frequent controls during the scan itself would disrupt the naturalistic experiment flow and efficiency. It is therefore challenging to experimentally monitor momentary attentional shifts (Bronkhorst 2015). To assess the influence of momentary shifts during sustained-attention tasks, future studies are warranted leveraging more controlled speech stimuli with systematic variations in the salience and task relevance of nontarget stimuli (Corbetta et al. 2008; Parmentier et al. 2014; Bronkhorst 2015).

In the current study, we find that attention strongly alters semantic representations in favor of the target stream across frontal and parietal cortices. This is in alignment with previous fMRI studies that found attentional response modulations in frontal and parietal regions (Hill and Miller 2010; Ikeda et al. 2010; Regev et al. 2019). That said, an important question is whether these modulations predominantly reflect enhanced bottom-up processing of attended speech or topdown attentional control signals (Corbetta and Shulman 2002; Seydell-Greenwald et al. 2014). Note that we find broad semantic representations across frontal and parietal cortices during the passive-listening experiment, in the absence of any demanding attentional tasks. Furthermore, a recent study suggests that various semantic categories are differentially represented in these higher-level cortical regions (Huth et al. 2016). Taken together, these findings imply that semantic modulations in frontoparietal regions can be partly attributed to bottom-up effects. Yet, it is challenging to disentangle bottom-up and topdown contributions in fMRI studies due to the inherently limited temporal resolution. Future studies are warranted to shed light on this issue by combining the spatial sampling capability of fMRI with high temporal resolution of electrophysiology methods (Çukur et al. 2013; de Heer et al. 2017).

\section{Conclusion}

In sum, our results indicate that attention during a diotic cocktail-party task with naturalistic stimuli gradually selects attended over unattended speech across both dorsal and ventral processing pathways. This selection is mediated by representational modulations for linguistic features. Despite broad attentional modulations in favor of the attended stream, we still find that unattended speech is represented up to linguistic level in the regions that overlap with the reorienting attention system. These linguistic representations of unattended speech might facilitate attentional reorienting and filtering during natural speech perception. Overall, our findings provide comprehensive insights on attentional mechanisms that underlie the ability to selectively listen to a desired speaker in noisy multispeaker environments. 


\section{Supplementary Material}

Supplementary material can be found at Cerebral Cortex online.

\section{Funding}

National Eye Institute (EY019684); European Molecular Biology Organization Installation (IG 3028); TUBA GEBIP 2015 fellowship; Science Academy BAGEP 2017 award.

\section{Notes}

The authors thank Jack L. Gallant, Wendy de Heer and Ümit Keleş for assistance in various aspects of this research. Conflict of Interest: None declared.

\section{References}

Alho K, Medvedev SV, Pakhomov SV, Roudas MS, Tervaniemi M, Reinikainen K, Zeffirio T, Näätänen R. 1999. Selective tuning of the left and right auditory cortices during spatially directed attention. Cogn Brain Res. 7:335-341.

Alho K, Vorobyev VA, Medvedev SV, Pakhomov SV, Roudas MS, Tervaniemi M, Näätänen R. 2003. Hemispheric lateralization of cerebral blood-flow changes during selective listening to dichotically presented continuous speech. Cogn Brain Res. 17:201-211.

Alho K, Vorobyev VA, Medvedev SV, Pakhomov SV, Starchenko MG, Tervaniemi M, Näätänen R. 2006. Selective attention to human voice enhances brain activity bilaterally in the superior temporal sulcus. Brain Res. 1075:142-150.

Alho K, Rinne T, Herron TJ, Woods DL. 2014. Stimulus-dependent activations and attention-related modulations in the auditory cortex: a meta-analysis of fMRI studies. Hear Res. 307:29-41.

Benjamini Y, Hochberg Y. 1995. Controlling the false discovery rate: a practical and powerful approach to multiple testing. $J$ R Statist Soc. 57:289-300.

Bizley JK, Cohen YE. 2013. The what, where and how of auditoryobject perception. Nat Rev Neurosci. 14:693-707.

Bregman AS. 1994. Auditory scene analysis: the perceptual organization of sound. London: MIT Press.

Broadbent D. 1958. Perception and communication. London: Pergamon Press.

Brodbeck C, Presacco A, Simon JZ. 2018a. Neural source dynamics of brain responses to continuous stimuli: speech processing from acoustics to comprehension. Neuroimage. 172:162-174.

Brodbeck C, Hong LE, Simon JZ. 2018b. Rapid transformation from auditory to linguistic representations of continuous speech. Curr Biol. 28:3976-3983.

Boulenger V, Hoen M, Ferragne E, Pellegrino F, Meunier F. 2010. Real-time lexical competitions during speech-in-speech comprehension. Speech Commun. 52:246-253.

Bronkhorst AW. 2015. The cocktail-party problem revisited: early processing and selection of multi-talker speech. Attent Percept Psychophy. 77:1465-1487.

Cherry EC. 1953. Some experiments on the recognition of speech, with one and two ears. J Acoust Soc Am. 25:975-979.

Corbetta M, Shulman GL. 2002. Control of goal-directed and stimulus-driven attention in the brain. Nat Rev Neurosci. 3:201-215.
Corbetta M, Patel G, Shulman GL. 2008. The reorienting system of the human brain: from environment to theory of mind. Neuron. 58:306-324.

Çukur T, Nishimoto S, Huth AG, Gallant JL. 2013. Attention during natural vision warps semantic representation across the human brain. Nat Neurosci. 16:763-770.

Da Costa S, van der Zwaag W, Marques JP, Frackowiak RS, Clarke S, Saenz M. 2011. Human primary auditory cortex follows the shape of Heschl's gyrus. J Neurosci. 31:14067-14075.

Da Costa S, van der Zwaag W, Miller LM, Clarke S, Saenz M. 2013. Tuning in to sound: frequency-selective attentional filter in human primary auditory cortex. J Neurosci. 33: 1858-1863.

Dale AM, Fischl B, Sereno MI. 1999. Cortical surface-based analysis - I: segmentation and surface reconstruction. Neuroimage. 9:179-194.

Davis MH, Johnsrude IS. 2003. Hierarchical processing in spoken language comprehension. J Neurosci. 23:3423-3431.

Davis MH, Johnsrude IS. 2007. Hearing speech sounds: top-down influences on the interface between audition and speech perception. Hear Res. 229:132-147.

de Heer WA, Huth AG, Griffiths TL, Gallant JL, Theunissen FE. 2017. The hierarchical cortical organization of human speech processing. J Neurosci. 37:6539-6557.

Degerman A, Rinne T, Salmi J, Salonen O, Alho K. 2006. Selective attention to sound location or pitch studied with fMRI. Brain Res. 1077:123-134.

Destrieux C, Fischl B, Dale A, Halgren E. 2010. Automatic parcellation of human cortical gyri and sulci using standard anatomical nomenclature. Neuroimage. 53:1-15.

Di Liberto GM, O'Sullivan JA, Lalor EC. 2015. Low-frequency cortical entrainment to speech reflects phoneme-level processing. Curr Biol. 25:2457-2465.

Ding N, Simon JZ. 2012a. Neural coding of continuous speech in auditory cortex during monaural and dichotic listening. $J$ Neurophysiol. 107:78-89.

Ding N, Simon JZ. 2012b. Emergence of neural encoding of auditory objects while listening to competing speakers. Proc Natl Acad Sci U S A. 109:11854-11859.

Ding N, Simon JZ. 2014. Cortical entrainment to continuous speech: functional roles and interpretations. Front Hum Neurosci. 8:311.

Ding N, Pan X, Luo C, Su N, Zhang W, Zhang J. 2018. Attention is required for knowledge-based sequential grouping: insights from the integration of syllables into words. J Neurosci. 38:1178-1188.

Driver J. 2001. A selective review of selective attention research from the past century. Brit J Psych. 92:53-78.

Elhilali M, Xiang J, Shamma SA, Simon JZ. 2009. Interaction between attention and bottom-up saliency mediates the representation of foreground and background in an auditory scene. PLoS Biol. 7:e1000129.

Evans S, McGettigan C, Agnew ZK, Rosen S, Scott SK. 2016. Getting the cocktail party started: masking effects in speech perception. J Cogn Neurosci. 28:483-500.

Friederici AD. 2011. The brain basis of language processing: from structure to function. Physiol Rev. 91:1357-1392.

Fritz JB, Elhilali M, David SV, Shamma SA. 2007. Auditory attention-focusing the searchlight on sound. Curr Opin Neurobiol. 17:437-455.

Gao JS, Huth AG, Lescroart MD, Gallant JL. 2015. Pycortex: an interactive surface visualizer for fMRI. Front Neuroinform. 9. doi: 10.3389/fninf.2015.00023. 
Gill P, Zhang J, Woolley SM, Fremouw T, Theunissen FE. 2006. Sound representation methods for spectro-temporal receptive field estimation. J Comput Neurosci. 21:5-20.

Goutte C, Nielsen FA, Hansen K. 2000. Modeling the hemodynamic response in fMRI using smooth fir filters. IEEE Trans Med Imag. 19:1188-1201.

Greve DN, Fischl B. 2009. Accurate and robust brain image alignment using boundary-based registration. Neuroimage. 48:63-72.

Griffiths TD, Warren JD. 2004. What is an auditory object? Nat Rev Neurosci. 5:887-892.

Gutschalk A, Dykstra AR. 2014. Functional imaging of auditory scene analysis. Hear Res. 307:98-110.

Hervais-Adelman AG, Carlyon RP, Johnsrude IS, Davis MH. 2012. Brain regions recruited for the effortful comprehension of noise-vocoded words. Lang Cognit Process. 27:1145-1166.

Hickok G, Poeppel D. 2004. Dorsal and ventral streams: a framework for understanding aspects of the functional anatomy of language. Cognition. 92:67-99.

Hickok G, Poeppel D. 2007. The cortical organization of speech processing. Nat Rev Neurosci. 8:393-402.

Hickok G, Poeppel D. 2016. Neural basis of speech perception. Neurobio Lang. 299-310.

Hill KT, Miller LM. 2010. Auditory attentional control and selection during cocktail party listening. Cereb Cortex. 20:583-590.

Hink RF, Hillyard SA. 1976. Auditory evoked potentials during selective listening to dichotic speech messages. Percept Psychophys. 20:236-242.

Huth AG, De Heer WA, Griffiths TL, Theunissen FE, Gallant JL. 2016. Natural speech reveals the semantic maps that tile human cerebral cortex. Nature. 532:453-458.

Ikeda Y, Yahata N, Takahashi H, Koeda M, Asai K, Okubo Y, Suzuki H. 2010. Cerebral activation associated with speech sound discrimination during the diotic listening task: an fMRI study. Neurosci Res. 67:65-71.

Jäncke L, Buchanan TW, Lutz K, Shah NJ. 2001. Focused and nonfocused attention in verbal and emotional dichotic listening: an FMRI study. Brain Lang. 78:349-363.

Jäncke L, Specht K, Shah JN, Hugdahl K. 2003. Focused attention in a simple dichotic listening task: an fMRI experiment. Cogn Brain Res. 16:257-266.

Jenkinson M, Smith S. 2001. A global optimization method for robust affine registration of brain images. Med Image Anal. 5:143-156.

Johnson JA, Zatorre RJ. 2005. Attention to simultaneous unrelated auditory and visual events: behavioural and neural correlates. Cereb Cortex. 15:1609-1620.

Kerlin JR, Shahin AJ, Miller LM. 2010. Attentional gain control of ongoing cortical speech representations in a "cocktail party". J Neurosci. 30:620-628.

Kidd G, Colburn HS. 2017. Informational masking in speech recognition. In: The Auditory System at the Cocktail Party. Vol 60. Cham: Springer, pp. 75-109.

Lavie N. 2005. Distracted and confused?: selective attention under load. Trends Cogn Sci. 9:75-82.

Levelt WJ. 1993. Speaking: from intention to articulation. Cambridge (MA): MIT Press.

Lewis JL. 1970. Semantic processing of unattended messages using dichotic listening. J Exp Psychol. 85:225-228.

Li Y, Wang F, Chen Y, Cichocki A, Sejnowski T. 2018. The effects of audiovisual inputs on solving the cocktail party problem in the human brain: an fmri study. Cereb Cortex. 28: 3623-3637.
Liberman AM, Mattingly IG. 1985. The motor theory of speech perception revised. Cognition. 21:1-36.

Lipschutz B, Kolinsky R, Damhaut P, Wikler D, Goldman S. 2002. Attention-dependent changes of activation and connectivity in dichotic listening. Neuroimage. 17:643-656.

Lyon R. 1982. A computational model of filtering, detection, and compression in the cochlea. IEEE Int Conf Acoust Speech Sign Proc. 7:1282-1285.

McDermott JH. 2009. The cocktail party problem. Curr Biol. 19:R1024-R1102.

Mesgarani N, Chang EF. 2012. Selective cortical representation of attended speaker in multi-talker speech perception. Nature. 485:233-U118.

Miller LM. 2016. Neural Mechanisms of Attention to Speech. In: Neurobiology of Language. San Diego: Academic Press, pp. 503-514.

Mitchell TM, Shinkareva SV, Carlson A, Chang KM, Malave VL, Mason RA, Just MA. 2008. Predicting human brain activity associated with the meanings of nouns. Science. 320:1191-1195.

Moray N. 1959. Attention in dichotic listening: affective cues and the influence of instructions. QJ Exp Psychol. 11:56-60.

Möttönen R, Dutton R, Watkins KE. 2013. Auditory-motor processing of speech sounds. Cereb Cortex. 23:1190-1197.

Nakai T, Kato C, Matsuo K. 2005. An fMRI study to investigate auditory attention: a model of the cocktail party phenomenon. Magn Reson Med Sci. 4:75-82.

Näätänen R, Kujala T, Winkler I. 2011. Auditory processing that leads to conscious perception: a unique window to central auditory processing opened by the mismatch negativity and related responses. Psychophysiology. 48:4-22.

Okada K, Rong F, Venezia J, Matchin W, Hsieh IH, Saberi K, Serences JT, Hickok G. 2010. Hierarchical organization of human auditory cortex: evidence from acoustic invariance in the response to intelligible speech. Cereb Cortex. 20: 2486-2495.

Osnes B, Hugdahl K, Specht K. 2011. Effective connectivity analysis demonstrates involvement of premotor cortex during speech perception. Neuroimage. 54:2437-2445.

O'Sullivan J, Herrero J, Smith E, Schevon C, McKhann GM, Sheth SA, Mehta AH, Mesgarani N. 2019. Hierarchical encoding of attended auditory objects in multi-talker speech perception. Neuron. 104:1195-1209.

Paltoglou AE, Sumner CJ, Hall DA. 2009. Examining the role of frequency specificity in the enhancement and suppression of human cortical activity by auditory selective attention. Hear Res. 257:106-118.

Parmentier FB, Turner J, Perez L. 2014. A dual contribution to the involuntary semantic processing of unexpected spoken words. J Exp Psychol. 143:38.

Petkov CI, Kang X, Alho K, Bertrand O, Yund EW, Woods DL. 2004. Attentional modulation of human auditory cortex. Nat Neurosci. 7:658-663.

Power AJ, Foxe JJ, Forde EJ, Reilly RB, Lalor EC. 2012. At what time is the cocktail party? A late locus of selective attention to natural speech. Eur J Neurosci. 35:1497-1503.

Pulvermüller F, Shtyrov Y. 2006. Language outside the focus of attention: the mismatch negativity as a tool for studying higher cognitive processes. Prog Neurobiol. 79:49-71.

Puschmann S, Steinkamp S, Gillich I, Mirkovic B, Debener S, Thiel CM. 2017. The right temporoparietal junction supports speech tracking during selective listening: evidence from concurrent EEG-fMRI. J Neurosci. 37:11505-11516. 
Puschmann S, Baillet S, Zatorre RJ. 2019. Musicians at the cocktail party: neural substrates of musical training during selective listening in multispeaker situations. Cereb Cortex. 29:3253-3265.

Puvvada KC, Simon JZ. 2017. Cortical representations of speech in a multitalker auditory scene. J Neurosci. 37:9189-9196.

Rauschecker JP, Scott SK. 2009. Maps and streams in the auditory cortex: nonhuman primates illuminate human speech processing. Nat Neurosci. 12:718-724.

Rauschecker JP. 2011. An expanded role for the dorsal auditory pathway in sensorimotor control and integration. Hear Res. 271:16-25.

Rämä P, Relander-Syrjänen K, Carlson S, Salonen O, Kujala T. 2012. Attention and semantic processing during speech: an fMRI study. Brain Lang. 122:114-119.

Regev M, Simony E, Lee K, Tan KM, Chen J, Hasson U. 2019. Propagation of information along the cortical hierarchy as a function of attention while reading and listening to stories. Cereb Cortex. 29:4017-4034.

Relander K, Rämä P, Kujala T. 2009. Word semantics is processed even without attentional effort. J Cogn Neurosci. 21:1511-1522.

Riecke L, Peters JC, Valente G, Kemper VG, Formisano E, Sorger B. 2017. Frequency-selective attention in auditory scenes recruits frequency representations throughout human superior temporal cortex. Cereb Cortex. 27:3002-3014.

Rinne T, Pekkola J, Degerman A, Autti T, Jääskeläinen IP, Sams M, Alho K. 2005. Modulation of auditory cortex activation by sound presentation rate and attention. Hum Brain Mapp. 26:94-99.

Rinne T, Balk MH, Koistinen S, Autti T, Alho K, Sams M. 2008. Auditory selective attention modulates activation of human inferior colliculus. J Neurophysiol. 100:3323-3327.

Rinne T. 2010. Activations of human auditory cortex during visual and auditory selective attention tasks with varying difficulty. Open Neuroimage. 4:187.

Rivenez M, Darwin CJ, Guillaume A. 2006. Processing unattended speech. J Acoust Soc Am. 119:4027-4040.

Rutten S, Santoro R, Hervais-Adelman A, Formisano E, Golestani N. 2019. Cortical encoding of speech enhances task-relevant acoustic information. Nat Hum Behav. 3:974-987.

Sabri M, Binder JR, Desai R, Medler DA, Leitl MD, Liebenthal E. 2008. Attentional and linguistic interactions in speech perception. Neuroimage. 39:1444-1456.

Scott SK, Rosen S, Wickham L, Wise RJ. 2004. A positron emission tomography study of the neural basis of informational and energetic masking effects in speech perception. J Acoust Soc Am. 115:813-821.

Scott SK, Rosen S, Beaman CP, Davis JP, Wise RJS. 2009a. The neural processing of masked speech: evidence for different mechanisms in the left and right temporal lobes. J Acoust Soc Am. 125:1737-1743.

Scott SK, McGettigan C, Eisner F. 2009b. A little more conversation, a little less action-candidate roles for the motor cortex in speech perception. Nat Rev Neurosci. 10:295-302.
Scott SK, McGettigan C. 2013. The neural processing of masked speech. Hear Res. 303:58-66.

Seydell-Greenwald A, Greenberg AS, Rauschecker JP. 2014. Are you listening? Brain activation associated with sustained nonspatial auditory attention in the presence and absence of stimulation. Hum Brain Mapp. 35:2233-2252.

Shinn-Cunningham BG, Best V. 2008. Selective attention in normal and impaired hearing. Trends Amplif. 12:283-299.

Shinn-Cunningham BG, Best V, Lee AK. 2017. Auditory object formation and selection. In: The Auditory System at the Cocktail Party. Vol 60. Cham: Springer, pp. 7-40.

Simon JZ. 2017. Human auditory neuroscience and the cocktail party problem. In: The auditory system at the cocktail party. Cham, Switzerland: Springer, pp. 169-197.

Slaney M. 1998. Auditory toolbox. Interval Research Corporation Technical Report. 10:1194.

Smith SM. 2002. Fast robust automated brain extraction. Hum Brain Mapp. 17:143-155.

Spence C, Driver J. 1997. Audiovisual links in exogenous covert spatial orienting. Percept Psychophys. 59:1-22.

Teder W, Kujala T, Näätänen R. 1993. Selection of speech messages in free-field listening. Neuroreport. 5:307-309.

Treisman A. 1960. Contextual cues in selective listening. QJ Exp Psychol. 12:242-248.

Treisman A. 1964. Monitoring and storage of irrelevant messages in selective attention. J Verb Learn Verb Behav. 3:449-459.

Vossel S, Geng JJ, Fink GR. 2014. Dorsal and ventral attention systems: distinct neural circuits but collaborative roles. Neuroscientist. 20:150-159.

Wikman P, Sahari E, Salmela V, Leminen A, Leminen M, Laine M, Alho K. 2021. Breaking down the cocktail party: attentional modulation of cerebral audiovisual speech processing. Neuroimage. 224:117365.

Wild CJ, Yusuf A, Wilson DE, Peelle JE, Davis MH, Johnsrude IS. 2012. Effortful listening: the processing of degraded speech depends critically on attention. J Neurosci. 32: 14010-14021.

Wood N, Cowan N. 1995. The cocktail party phenomenon revisited: how frequent are attention shifts to one's name in an irrelevant auditory channel? J Exp Psychol Learn Mem Cogn. 21:255-260.

Woods DL, Stecker GC, Rinne T, Herron TJ, Cate AD, Yund EW, Liao I, Kang X. 2009. Functional maps of human auditory cortex: effects of acoustic features and attention. PLoS One. 4:e5183.

Woods DL, Herron TJ, Cate AD, Yund EW, Stecker GC, Rinne T, Kang X. 2010. Functional properties of human auditory cortical fields. Front Syst Neurosci. 4:155.

Yuan J, Liberman M. 2008. Speaker identification on the SCOTUS corpus. J Acoust Soc Am. 123:3878.

Zion Golumbic EM, Ding N, Bickel S, Lakatos P, Schevon CA, McKhann GM, Goodman RR, Emerson R, Mehta AD, Simon JZ. 2013. Mechanisms underlying selective neuronal tracking of attended speech at a 'cocktail party'. Neuron. 77:980-991. 